\title{
Wound Closure and Care in Oral and Maxillofacial Surgery
}

\author{
Ravi Veeraraghavan
}

\subsection{Wound Management}

Management of soft tissue wounds needs a careful assessment of the wound, a good understanding of wound types and healing as well as adequate knowledge and skill for wound care. The various steps in wound care include cleansing and debridement, hemostasis, tetanus immunization and antibiotic medication and wound closure.

Wound closure can be accomplished in diverse ways, but suturing remains the mainstay. A wide variety of materials are available for wound suturing. One has to choose the right material and technique depending on the wound type and closure needs. Alternate techniques such as staples, tapes and adhesives are fast gaining popularity.

Wounds are treated by

1. Cleansing and debridement,

2. Haemostasis,

3. Tetanus immunisation,

4. Wound dressings,

5. Wound closure,

6. Antibiotic medication.

\subsection{Wound Closure}

Artificial closure of the wounds is required if significant connective tissue is exposed. Wound closure leads to faster wound healing with reduced complications. Even though suturing is the mainstay of wound closure, other alternatives are fast gaining popularity.

Electronic Supplementary Material The online version of this chapter (https://doi.org/10.1007/978-981-15-1346-6_11) contains supplementary material, which is available to authorized users.

R. Veeraraghavan $(\bowtie)$

Department of Oral and Maxillofacial Surgery, Amrita School of

Dentistry, Ernakulam, India

\subsubsection{Purposes of Wound Closure}

Before going into the intricacies of material selection and techniques of wound closure, one needs to be clear about the actual need for artificially closing a wound. The main indication for wound closure is significant exposure of connective tissue. In general, there are five reasons behind the concept of wound closure.

\section{Healing by Primary Intention}

This is the most important purpose of wound closure. The open wounds, which are likely to heal by secondary intention, are made to heal in a 'more' primary manner by the intervention of wound closure. This hastens the healing process and also reduces scar formation, with resultant advantages in aesthetic and functional facets.

2. Coverage of Deep Tissues

When the epithelium is breached and connective tissue is bared, it exposes the inner tissues to mechanical and biological threats. This threat becomes even more serious when deeper structures such as the viscera or bone is exposed.

3. Prevent Contamination

An open wound is under constant attack by the pathogenic microorganisms, more so in the oral cavity. Such relentless contamination is likely to lead to a full-blown infection and/or delayed healing. Sooner the wound is closed, the lesser the risk of major contamination.

\section{Haemostasis}

This is not one of the primary purposes of wound closure. But sutures may help in impeding blood loss by preventing mechanical disturbance to the clot.

\section{Prevention of Dead Space}

If only the superficial cutaneous sutures are placed over a deep wound, a dead space can result beneath the surface. This condition usually leads to wound breakdown and/or wound infection. Dead space is prevented by closing the deep tissues in multiple layers. 


\subsection{Wound Suturing}

Suturing is the process of wound closure by holding the edges of the wound together using a thread. This thread, called a 'suture', is used to approximate the wound edges together and to hold them in position till the tissue healing is sufficiently advanced to maintain themselves in the corrected anatomic position and alignment to each other. The surgeon uses a 'suture needle' to pass the thread through the tissues on either side of the wound so as to hold the separated edges in the proper position.

\subsubsection{Suturing Instruments}

Apart from the suture thread and needle, some instruments are used to perform the suturing process. Commonly used instruments include a needle holder, a tissue holding forceps and a pair of scissors.

\subsubsection{Needle Holder}

The needle holder (needle driver) is the primary instrument used for suturing. Similar to a haemostat, it is used to grasp and manipulate the suture needle to which the thread is attached. A needle holder has three parts — a pair of beaks, a joint ('pivot') and a pair of handles with rings. Many designs are available, examples being Webster, Halsey and MayoHegar. Most designs have a serrated or cross-hatching pattern of teeth for the beaks. This helps to grip the needle securely without damaging it. A locking mechanism similar to haemostats is present to lock the needle grasp.

The classical way to hold the instrument is to engage the handle-rings with the thumb and the fourth finger. The middle finger is used for support and the index finger is used to direct or orient the instrument in the required direction. Alternatively, while suturing on tough tissues, one may be 'palmed', i.e. may be held against the palm with fingers around. While palming the needle holder, some surgeons still rest the fourth finger lightly in the handle-ring.

\subsubsection{Tissue Holding Forceps}

The pickup forceps are used to delicately handle and manipulate the wound edge which is being sutured. The finetoothed Adson forceps is the workhorse for most interior and cutaneous wound closures. The forceps is held in the nondominant hand and is used to grasp, lift and evert the wound edge so that the surface is made more amenable to a perpendicular needle entry. Fine skin hooks may also be used for the same purpose.

\subsubsection{Suture Cutting Scissors}

The suture cutting scissors is used to cut the suture thread tags after the knot is tied. It is also used to cut the thread during suture removal.
These scissors should be differentiated from scissors used for cutting tissue. Many of them have specifically designed blades and tips, which are uniquely shaped to lift and grasp up the handing tags or the tied suture thread away from the tissue and to cut it safely without injuring the tissues. Short and long versions are available. The tip may be straight or curved and may have a sharp slot for the thread to be cut. Some manufacturers have incorporated a cutting component in the needle holder itself, using which the surgeon can cut the suture tag without switching instruments.

\subsubsection{Suture Needles}

The suture needles help to pass the suture threads through tissues. In ancient days, needles were made of natural materials such as bones, ivory, horns, wood or thorns. In fact, eyed needles from as early as 30,000 AD have been unearthed. Later, metals such as silver, copper and aluminium bronze were used. The modern needles are diligently shaped and contoured as per use and are smooth and strong so that they withstand the stresses of suturing and least traumatic to tissues at the same time.

The suture needle has three parts: the tip, the body and the suture attachment. Made of stainless steel or carbon steel, they are thin, smooth and sharp, designed to pass through tissues with ease (Fig. 11.1).

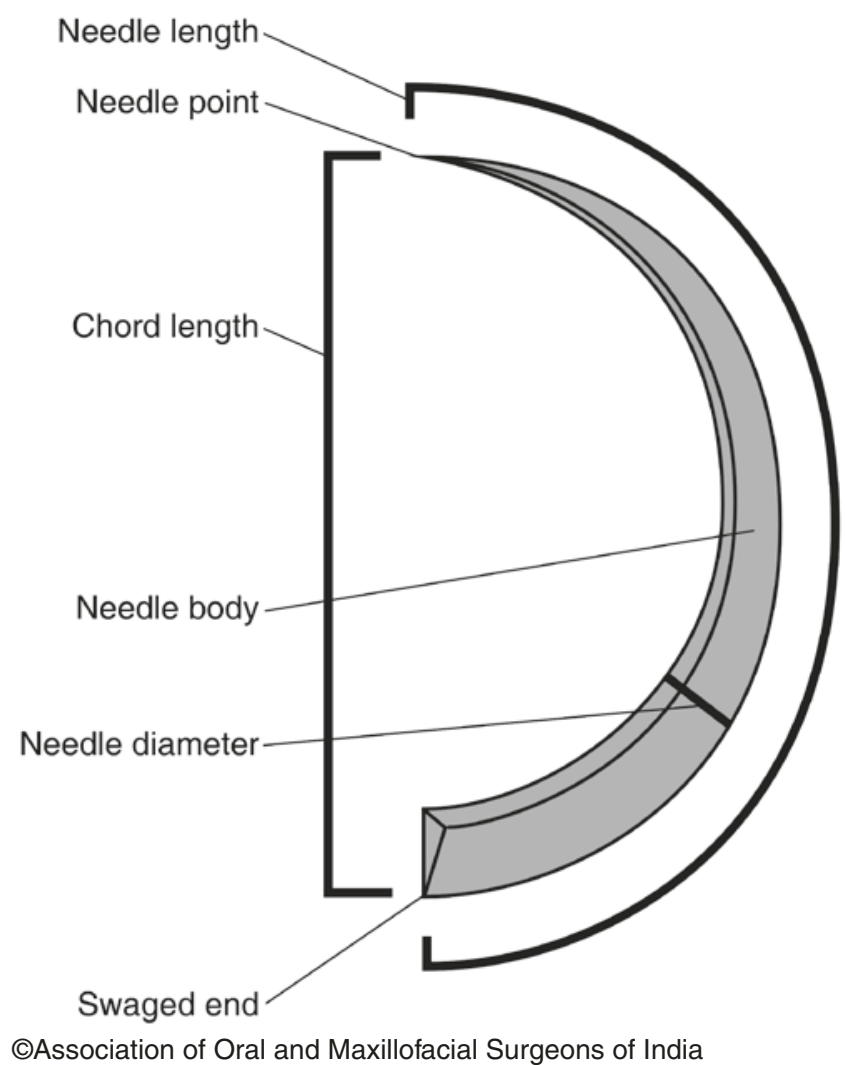

Fig. 11.1 Parts of suture needle 
Fig. 11.2 Needle shapes/ curvature

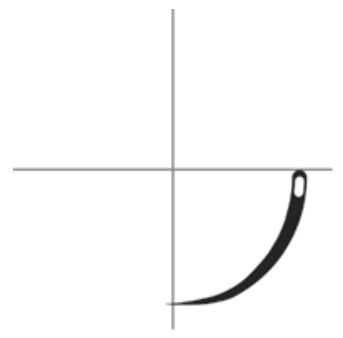

1/4 Circle

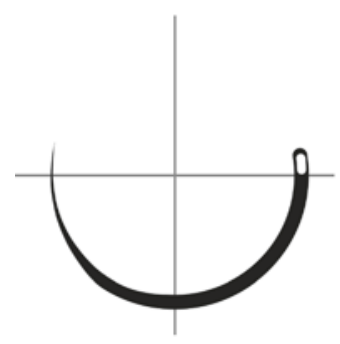

5/8 Circle

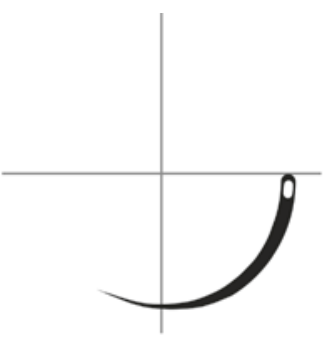

3/8 Circle

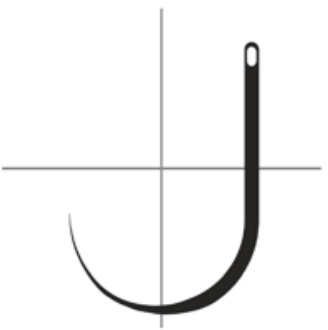

$J$ shape

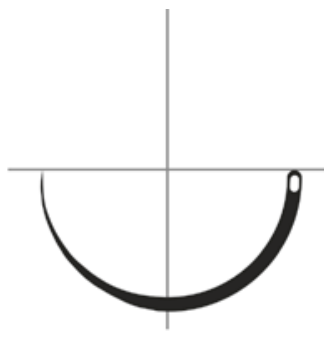

1/2 Circle

CAssociation of Oral and Maxillofacial Surgeons of India

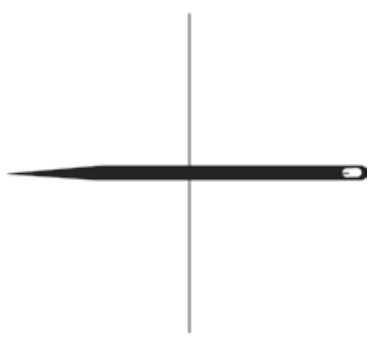

Straight

\subsubsection{Needle Shape}

The suture needles are usually curved as an arc of a circle. Straight needles, though available, have very limited practical applications. The curvature is described as the span of the arc, in terms of $1 / 4,3 / 8,1 / 2$ and $5 / 8$ of a circle. Most of the regular suturing in oral and maxillofacial surgery is done with half circle needles. A smaller arc such as $3 / 8$ can be used for suturing flat skin surfaces. Longer arc, i.e. $5 / 8$ circle, needles are used in narrow tunnelled surgical fields such as in cleft palate or inside the nose (Fig. 11.2).

The cross section and the tip of the needles can be widely varied. Depending on these aspects, the suture needles can be.

1. Round bodied,

2. Conventional cutting (Fig. 11.3) or

3. Reverse cutting.

Round bodied needles are generally used to suture viscera and other internal structures such as muscle and fascia. It can also be effective on non-keratinised mucosa. The tip of the round-bodied needle can be blunt or sharp. The blunt tip needle is specifically used in the inner abdominal wall and in friable tissues. The sharp tip is used for most other purposes. But even the sharp tip is hard to pass across the skin and keratinised mucosa. The shape of the 'cutting' needle overcomes this problem. In the cutting needles, the needle cross section is a triangle, with the inner curve side being pointed

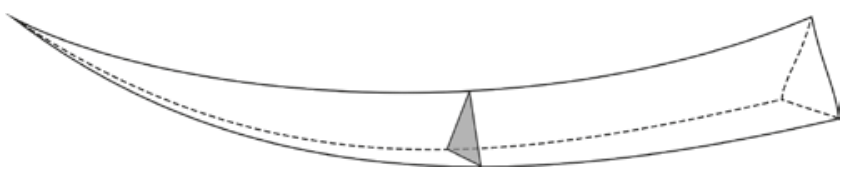

CAssociation of Oral and Maxillofacial Surgeons of India

Fig. 11.3 Needle cross section of a conventional cutting needle

and the outer convex side flat. This shape enables the needle to 'cut' through the dense tissues to make the needle passage easier. But the cutting design presented a new problem. Even under minimum tension, the needle behaved like a knife, and the sharp edge often tended to lacerate the tissue resulting in a 'cut-through' of the wound edge. This was solved by the reverse-cutting design, wherein the inner curve is flat and the outer curve is pointed. This helps to retain the cutting nature while avoiding the risk of wound edge laceration. In oral and maxillofacial surgery, the tissues encountered may be keratinised (skin, gingiva and palate) or non-keratinised (buccal mucosa, floor of mouth and lips). Mostly, reverse-cutting needles are now being used since they are well-suited to pass through all oral tissues without causing inadvertent tears.

\subsubsection{Needle Size}

The suture needle size generally depends on the size of the suture. In general, smaller the suture size, smaller the needle. It is important to recognise the various dimensions of a needle. 
The 'needle length' refers to the end-to-end measurement along the curve of the needle. The straight line distance from the tip to the other end is called 'chord length'. The chord length determines the bite-width of the needle in the tissues. The bite-depth into the tissue is defined by the "chord diameter' which is perpendicular from the chord length to the centre of the needle body. Needle diameter and needle radius are dimensions which refer to the cross-sectional thickness and not to the needle curve.

\subsubsection{Eyed vs Swaged Needles}

The eye of the needle is a small hole at one end for attaching the suture thread. This necessitates an increase in the needle thickness at the attachment end, increasing the risk of potential tissue trauma. The suture is passed through the eye, and may then be tucked or tied for better retention. Both tucking and tying further increases the tissue drag and causes additional trauma. The attached suture now follows the needle through the tissues. The eyed needles are cheaper and can be reused after resterilisation.

Modern suture needles do not have an eye. Instead, the suture thread is sealed inside the needle for a permanent attachment. This mechanism, called 'swaging', reduces the tissue trauma significantly as the needle diameter is not increased for thread attachment. Further, only a single thread strand passes through the tissue at all times. So these needles are often called 'atraumatic needles'. The swaged needle with thread is available as a sterile pack, and cannot be reused.

\subsection{Suture Thread}

Many different types of suture threads are available for use. Each type has its specific benefits and indications for application (Table 11.1).

A basic classification divides them into 'natural' and 'synthetic'. Suture materials sourced from natural sources include catgut, steel and silk. Synthetic plastic materials such as nylon, polypropylene and polymers of glycolic acid, lactic acid, etc., have become popular in recent decades.

Suture materials can also be classified as absorbable and non-absorbable. Absorbable sutures hold the tissues together till they have healed sufficiently to withstand normal stress, and are then absorbed by the tissues where they are embed-

Table 11.1 Suture material classification

\begin{tabular}{|c|c|}
\hline $\begin{array}{l}\text { Basis of } \\
\text { classification }\end{array}$ & Types \\
\hline Source & Natural, synthetic \\
\hline Absorbability & Absorbable, non-absorbable \\
\hline Thread size & $\begin{array}{l}3,2,1,0,1-0,2-0, \\
3-0 \ldots \ldots \ldots \ldots \ldots \ldots \ldots \ldots .11-0\end{array}$ \\
\hline Filament type & Monofilament, multifilament (braided) \\
\hline
\end{tabular}

ded. Non-absorbable sutures, on the other hand, are not absorbed, and they should be removed after the initial stabilisation of the wound edges has occurred. In reality, most or all natural materials will get absorbed by the body in due course of time. Thus, for clinical practice, a suture is considered non-absorbable if it retains its tensile strength in tissues for more than 60 days.

According to the filament type, the sutures may be monofilament or multifilament. A monofilament suture is essentially a single strand of fibre. This simple structure enables these sutures to pass through tissues with least resistance. Though this makes it the material of choice in delicate tissues such as the vascular tissues, there are some notable drawbacks. The handling and knot-tying are generally more taxing, and the knot has less friction to keep its position. In addition, the cut edges tend to be stiff leading to probable irritation of movable and delicate regions like the tongue and buccal mucosa.

The multifilament sutures are made of multiple fibre strands, which are either coated or braided together to make one fine thread. The process of braiding bestows a higher tensile strength as well as better pliability and flexibility. This improves the handling characteristics and the ease of knottying. They also better resist the tendency of the knot to untie itself. The cut edges are usually smooth and soft, and are not irritating to oral tissues. The disadvantages of multifilament sutures include the higher risk of harbouring pathogens in its structure and the tendency to 'wick' the oral fluids into deeper tissues through the suture track. This, in turn, can lead to occurrence to unaesthetic suture tracks visible on the skin.

\subsubsection{Suture Thread Size}

The suture thread is available in different thicknesses. The thickness is usually mentioned as 'number of zeroes'. This system follows the U.S. Pharmacopeia, and has gained wide acceptance. The most commonly used thread size for intraoral use is mentioned as 3-0 or 000 size. Sutures are available from size 6 to $11-0$ (Table 11.2).

\section{Attributes of an Ideal Suture Material}

1. Sterile or sterilisable,

2. Good handling characteristics,

3. Ensure a secure knot,

4. Adequate tensile strength,

5. Retention of strength during the healing phase,

6. Rapid absorption after healing.

7. Stay inert in tissues without causing antigenicity or other adverse tissue reaction. 
Table 11.2 Suture sizes-U.S. Pharmacopeia designation and equivalent metric measurements

\begin{tabular}{|l|l|l|l|l|l|l|l|l|l|l|l|l|l|l|l|l|l|l|}
\hline USP designation & $11-0$ & $10-0$ & $9-0$ & $8-0$ & $7-0$ & $6-0$ & $5-0$ & $4-0$ & $3-0$ & $2-0$ & 0 & 1 & 2 & 3 \\
\hline Diameter $(\mathrm{mm})$ & 0.01 & 0.02 & 0.03 & 0.04 & 0.05 & 0.07 & 0.1 & 0.15 & 0.2 & 0.3 & 0.35 & 0.4 & 0.5 & 0.6 \\
\hline
\end{tabular}

It is beyond the purview of this chapter to go into the details of all the types of suture materials available in the market, chemical structure, method of resorption, qualities, indications, advantages, etc. Details of few suture materials widely used in oral and maxillofacial surgery are given below.

\subsubsection{Natural Absorbable Sutures (Absorbed by Proteolysis)}

The absorbability characteristics of many natural materials had been recognised for millennia. All absorbable natural suture materials contain predominantly collagen. Over the years, they have progressively fallen out of favour due to the risk of antigenicity and adverse tissue reaction [1].

\subsubsection{Catgut}

Catgut has been the most popular material in this group. Its use has been prevalent at least from the time of Galen of Pergamon (200 AD). This material is made of tissue taken from the submucosal layer of sheep intestine or from the serosal (adventitial) layer of the cattle small intestine intima. The collagen strands are twisted together and the resultant thread is precision-ground to form a monofilament. Catgut has long been a popular fibre to make string instruments and tennis racquets. For many centuries, catgut (surgical gut) had been the only absorbable suture material available. The absorption is by proteolytic biodegradation brought about by proteolytic enzymes.

Performance-wise, catgut has good flexibility but relatively poor tensile strength, poor knot stability and high tissue reactivity. Full tensile strength remains only for 7 days.

\subsubsection{Chromic Catgut}

It has been found that many poor characteristics of catgut can be overcome by impregnating the suture material with chromic salts. This modified product, called chromic catgut, has higher tensile strength and delayed absorption time. Chromic catgut retains its maximum tensile strength for about 2 weeks. It also has reduced tissue reaction as compared to plain gut.

Manufacturers package catgut sutures soaked in the disinfectant isopropyl alcohol solution to retain the flexibility and to increase shelf life. This necessitates rinsing of the suture in sterile saline to remove the irritant alcohol before use. Some manufacturers use a glycerine coating on chromic catgut to do away with the alcohol in packaging. Glycerine- coated chromic gut is smoother and thicker and has better handling characteristics.

\subsubsection{Natural Non-absorbable Sutures}

These are the oldest suture materials known to mankind. People tended to use any threaded material known to them for holding the wound edges together. Non-absorbable natural materials include cotton, linen, steel and silk.

\subsubsection{Cotton and Linen}

Cotton and linen sutures primarily contain cellulose polymer. Both the materials get absorbed in due course, but are considered non-absorbable because of delayed absorption time. Cotton is sourced from the hair of the cotton seed while linen is made from flax. The fibres are twisted to form a suture. It has good tensile strength, but there is moderate tissue reaction. Handling is average but knot-holding is good. Linen has the advantage that it gains tensile strength when wet.

Cotton and linen have largely fallen out of favour because of adverse tissue reactions and the high 'wicking' effect which causes seepage of fluids into the suture track.

\subsubsection{Silk}

Silk has been a well-known textile material from fourth millennium BC. It is produced by the silkworm larvae to form the cocoon. Natural silk is a protein fibre, the main components being fibroin and sericin. Fibroin forms the basic structure and contributes to the tensile strength. Sericin is a gum layer which holds the strands together. The silkworm larvae are cultivated and once they start pupating, the cocoons are dissolved in boiling water to extract the individual fibres which are fed into the spinning reel. To make it suitable as a medical product, the sericin protein component is later removed by a degumming process.

Silk is the most widely used natural non-absorbable suture material. Surgical silk is made from the larvae of the silkworm Bombyx Mori. The silk suture consists of a group of strands braided around a core and has a wax or silicon coating. It is usually dyed black in colour for better visibility.

The most impressive property of the silk suture is the ease of handling. It is extremely pliable and smooth and has good knot-holding capability. But the tensile strength is pretty low. The other drawbacks include high levels of tissue friction, capillary action and tissue inflammatory response. The wax coating helps to counteract all these negative attributes. 
Silk is now not considered an appropriate material for cutaneous suturing, except on specific sites such as eyelids and lips. It is very often used for ligating blood vessels and for hitching drains. It is the most popular material used in dentistry. Its soft and pliable nature makes it suitable for use in oral mucosa which is mobile and wet.

\subsubsection{Synthetic Non-absorbable Sutures}

\subsubsection{Polyamide/Nylon (Ethilon, Dermalon)}

Nylon belongs to the first generation of commercially successful synthetic thermoplastic polymers, having started production in 1927. It is made of repeated units linked by aliphatic or aromatic amide links (polyamide). It has been a very versatile material and has been used as fabric, fibres, films, coatings and moulded shapes for wide applications in diverse fields.

Nylon surgical sutures were introduced in 1940. Monofilament nylon sutures are very popular for cutaneous suturing. The main advantages are their high tensile strength, exceptional elasticity and low tissue reaction. The elasticity helps the material to accommodate tissue swelling and maintain wound edge apposition. The nylon sutures are dyed black in colour.

The biggest shortcoming of this material is its shape memory, which negatively affects its knot-tying and knotholding properties. Often, one needs 3-4 knots to hold a stitch in place. Also, the monofilament may be stiff. Multifilament nylon sutures are available with increased pliability and handling features. Addition of fluid (alcohol) in the package reduces the shape memory and improves pliability.

\subsubsection{Polypropylene (Prolene, Surgipro)}

Polypropylene is another thermoplastic polymer used as a non-absorbable suture material. It is produced by chaingrowth polymerisation of the monomer propylene. It is pigmented blue to enhance visibility, hence the name polypropylene blue.

Similar to nylon, polypropylene has a very high tensile strength, excellent elasticity and minimal tissue reaction. It can extend up to $30 \%$ without breakage, making it highly suitable for suturing cutaneous wounds. The shape memory is also similar to nylon and can contribute to knot slippage. This feature, though, is advantageous in subcuticular suturing since it slides out smoothly during suture removal.

The excellent mechanical properties and inert nature have made polypropylene the material of choice in stressful sites. It is widely used in the management of hernia and vaginal prolapse, often in the form of a mesh in addition to sutures.

\subsubsection{Polyester (Ethibond, Surgidac, Dacron)}

Polyester is a general term used for any organic polymer which has an ester functional group in the main chain. More specifically, the term is used for the material polyethylene terephthalate. It is a type of petroleum-based plastic, made by mixing ethylene glycol and terephthalic acid. Because of its durability, cleanability, anti-wrinkle and quick-drying characteristics, it swiftly made a name in the textile industry as a reliable material.

Polyester is a non-absorbable, braided, surgical suture with high tensile strength and low tissue reactivity. The braided nature adds to enhanced handling, knot-tying and retentivity. Thus, it combines the positive features generally attributed to monofilaments and multifilaments. It is thus the suture of choice in cardiovascular surgery, prosthetic implants and facelifts.

Polyester braided sutures are usually dyed green in colour. They may be coated or uncoated. The uncoated variant has a rough surface which produces drag in the tissues. The coating is made of PTFE or polybutylate.

\subsubsection{Polybutester (Novafil, Vascufil)}

Polybutester is a relatively new thermoplastic material with unique stress-strain properties. It is a copolymer comprised of polybutylene terephthalate and poly teramethylene ether glycol, and is coated with polytribolate. The polybutester monofilament sutures are designed to have high strength, elasticity and pliability. The flexibility and lack of memory allow it to be handled with ease resulting in a high knot security. The unique features have made this a popular material for abdominal wound closure.

The elasticity of polybutester is phenomenal. It can stretch $50 \%$ of its length at initial loads. It has a biphasic expansion curve wherein it expands well in response to initial stress and maintains pressure without cutting the tissue, and at the same time withstanding creep by not undergoing permanent deformation even under constant pressure.

\subsubsection{Polytetrafluroethylene (Gore-Tex, Cytoplast, Coreflon, Teflon)}

Polytetrafluroethylene (PTFE) is a synthetic material which has found extensive application globally. It is a fluoropolymer of tetrafluoroethylene $\left(-\mathrm{CF}_{2}-\mathrm{CF}_{2}-\right)$, made by free-radical polymerisation of monomer units. PTFE is a strong, tough, waxy, non-flammable material popular for its non-stick properties.

PTFE suture is considered as the ideal material for oral surgeries, especially for dental implant surgeries. It is inert, non-absorbable and monofilament in nature. The strong fluoro-carbon bond is thought to be the reason for its inertness. Unlike other synthetic monofilament sutures, PTFE is smooth, supple and soft. The cut ends cause no 
irritation to delicate oral tissues. It has no shape memory. It is well tolerated in the oral cavity and has excellent handling, knot-tying and knot-holding abilities. Thus, it has most of the positive attributes of braided sutures, at the same time avoiding the risk of bacterial contamination by wicking effect.

\subsubsection{Stainless Steel}

Surgical stainless steel non-absorbable surgical suture is composed of $316 \mathrm{~L}$ austenitic stainless steel. It can be monofilamentous or multifilamentous. The obvious advantages of stainless steel sutures are strength and low tissue reaction. Its drawbacks include very poor flexibility that makes it highly demanding in suturing skills. Incorrect technique can cause an excessive pull or tear on the tissues resulting in necrosis of wound edges. Barbs at the end can cause glove punctures and trauma to adjacent tissues.

Stainless steel suture is used in sternal closure and in orthopaedic procedures involving cartilage and tendon repair. It is also sparingly used for abdominal wound closure and hernia repair.

\subsubsection{Synthetic Absorbable Sutures (Absorbed by Hydrolysis)}

Till polyglycolic acid sutures were introduced in the 1970s, all absorbable sutures were natural. The synthetic absorbable sutures are all polymers based on glycolic acid, 1-lactic acid, paradioxanone, trimethylene carbonate and e-caprolactone [2]. They are sterilised either by ethylene oxide gas or by gamma radiation. These polymers have definite advantages over chromic catgut in clinical use. They are much stronger, evoke minimal tissue reaction, stay longer before absorption and leave no reactive changes after they are resorbed. They are absorbed typically by hydrolysis reaction which breaks the polymer chains. The hydrolysis end products are $\mathrm{CO}_{2}$, $\mathrm{H}_{2} \mathrm{O}$ and the monomer.

\subsubsection{Polyglycolic Acid (Dexon, PolySyn, PGA)}

Polyglycolic acid suture, introduced in the early 1970s, was the first absorbable synthetic suture material. It is braided homopolymer of glycolic acid. The uncoated version is beige in colour while the polycaprolate-coated product may be undyed or dyed green, violet or bicoloured. When compared to catgut, the tensile strength and knot security are excellent. It retains $65 \%$ of its tensile strength after 2 weeks, by which time catgut would have lost all its strength.

The polylycolic acid soon became very popular but fell out of favour later as better products were developed in due course of time.

\subsubsection{Polyglactin 910 (Vicryl, Polysorb)}

Polyglactin 910 is a synthetic heteropolymer consisting of $90 \%$ glycolic acid and 10\% lactic acid. Introduced in 1974, this is a multifilamentous, braided suture with a lubricant coating of polyglactin 370 (30:70 ratio) and calcium stearate. The final product is usually dyed violet in colour, but an undyed beige version is also available. Polyglactin 910 is among the most popular absorbable sutures used for surgical wound closure today.

The main advantages of polyglactin over polyglycolic acid include consistently higher residual tensile strength and faster absorption. The absorption happens between 40 and 70 days. The coating ensures smooth passage. Since the coating is made of similar material, the risk of flaking is very low. Calcium stearate used in the coating is an absorbable organic lubricant. The shelf life of polyglactin is as high as 5 years.

As the material became very popular and widely used, manufacturers began to bring out modified products providing specific benefits. One of them is a monofilament version which does not require a coating. Another one is an 'antibacterial suture' with embedded triclosan, which is said to be very effective in preventing surgical site infections. Yet another useful modification is 'rapidly absorbing polyglactin' (Vicryl Rapide/Velosorb Fast). By treating the coating with $\gamma$-radiation, it is made to lose strength by second week and is fully absorbed by sixth week. This variant is widely used in oral surgery, where faster resorption is desired

\subsubsection{Polydioxanone (PDS, PDO)}

Polydioxanone was the first monofilament suture available of large size (larger than 3-0). It is a polyester product and is synthesised through the ring opening of the monomer paradioxanone (1, 4-dioxan 2-one). As a monofilament suture, it has much less drag through the tissues than polyglactin or polyglycolic acid. Since it retains its tensile strength over a long span of time $(80 \%$ strength at 2 weeks and $60 \%$ after 6 weeks), it is considered a better alternative to polyglactin for suturing of fascia.

A modification called PDS II is chemically similar but is annealed above melting temperature to soften the external surface, imparting improved flexibility to the final product.

\subsubsection{Poliglecaprone 25 (Monocryl/Biosyn/ Petcryl Mono/Monoglyde)}

Often seen as the monofilament alternative to polyglactin, poliglecaprone is a segmented block polymer consisting of $75 \%$ glycolide and $25 \% \varepsilon$-caprolactone. It is available as an undyed or a violet dyed version. The key feature that differentiates poliglecaprone from other monofilament sutures is the high level of pliability and handling properties. This is 
achieved through the formation of an interim soft polymer chain 'pre-polymer' which is high in caprolactone. In the ensuing stages of manufacturing, more glycolide is added to supplement hard segments to the pre-polymer. In this way, we get a final product with high tensile strength without compromising on the pliability.

The tensile strength of the undyed poliglecaprone suture degrades to $50 \%$ in 1 week and $30 \%$ in the second week. The dyed version retains $70 \%$ strength after 1 week. Complete absorption by hydrolysis happens in about 100 days.

\subsubsection{Polyglyconate (Maxon)}

This copolymer has a molar ratio of $64 \%$ glycolic acid and $36 \%$ trimethylene carbonate. It is an uncoated monofilament which may be undyed or dyed dark green. Polyglyconate has good handling properties. But its main advantage is the retention of tensile strength over a long time span. It retains at least $50 \%$ of its strength 4 weeks after implantation, making this an excellent choice in situations where long-term retention is needed. The slow absorption makes it relatively unsuitable for subcuticular sutures, since the dyed suture may be visible under the surface.

\subsubsection{Glycomer 631 (Biosyn)}

It is a synthetic absorbable coated monofilament polyester suture. It is a tri-block copolymer and contains glycolide $(60 \%)$, trimethylene carbonate $(26 \%)$ and p-dioxanone $(14 \%)$. It may be undyed or dyed violet. It has high flexibility, low memory and minimal tissue reactivity.

It passes through the tissues easily but has poor knotholding capability. Degradation and absorption are similar to polyglactin. Full absorption is complete in around 100 days.

\subsubsection{Polyglytone 6211 (Caprosyn)}

Polyglytone 6211 is the only suture available with four different monomers in its core structure. It is a polyester copolymer of glycolide, caprolactone, trimethylene carbonate and lactide in the ratio $6: 2: 1: 1$. It is a monofilament, uncoated, absorbable suture which is undyed or dyed violet.

Polyglytone 6211 is a suture for the short-term approximation of tissues. Because of its quick disintegration, it offers an inert alternative to catgut that can evoke inflammatory response. Its strength decreases to 50-60\% at 5 days and to $20-30 \%$ at 10 days post-implantation.

\subsection{Knot-Tying}

The suture knot-tying can be accomplished by using an instrument (usually the needle holder) or using hands. Three main techniques are described.

\subsubsection{Two-Handed Tie}

The two-handed tie is cumbersome and is not routinely used. The suture is tied together by holding one tag in each hand and intertwining them.

\subsubsection{One-Handed Tie}

The one-handed tie is the most popular hand-tie method. It is quicker and can be accomplished in a smaller space. One end of the suture thread tag ('the long end') is held with thumb and forefinger of the dominant hand. The other side tag ('the short end') is placed a bit distal to the first tag, running in the same direction. The middle finger is folded and is used to guide the short end around the long end, and then the two tags are pulled away.

\subsubsection{Instrument Tie}

Instrument tie is the most popular technique for tying a suture knot. The long end is wrapped around the needle holder. Then the needle holder beaks are opened to grasp the short end, which is then pulled to form a tie. One such tie is called a 'throw'.

\subsection{Suture Knots}

The suture thread tags are intertwined to form a knot. The knot should be firm and tight, and should not lie on the wound/incision line. It is important that the knot stays tight and maintains strength during the healing phase. Knot slippage or breakage before healing can be detrimental to the wound. In general, knot security is higher for braided and uncoated sutures because of higher friction coefficient.

The knots placed for the surgical suturing are of simple design. Those employed commonly are the square knot, surgeon's knot and granny's knot. These three variants are very similar to each other and differ from each other only in minor aspects.

\subsubsection{Square Knot/Reef Knot}

A square knot is among the simplest of the knot designs. It involves a simple intertwining of the two threads. This 'halfhitch' knot (one 'throw' around the instrument) need to be complemented with an additional similar throw to make it secure. Preferably the second hitch should be in the opposite 
direction, i.e. if the first throw is in clockwise direction, the second one should be in anticlockwise orientation.

The classical square knot formed by a clockwise and a counterclockwise hitch can be further complimented by more similar hitches in alternatively changing directions.

\subsubsection{Surgeon's Knot}

A surgeon's knot is a minor modification of the square knot in which the initial intertwining is doubled. This is accomplished by doubling the first throw, by doing two turns of the thread around the instrument. This is followed by a regular second throw in the opposite direction.

\subsubsection{Granny's Knot}

In the granny's knot, two initial throws are placed in the same direction, followed by a third throw in the opposite direction.

\section{Principles of Wound Suturing}

Regardless of the specific technique, some basic principles are to be followed while performing a suturing procedure. It is to be remembered that these are only general principles, and can be modified or rejected in specific situations depending on the clinical context.

1. The suture needle is to be held with the needle holder about $34^{\text {th }}$ of the distance from the needle tip.

2. The selected suture size should be the smallest possible which will hold the wound edges securely.

3 . The needle tip should enter the tissue perpendicular to the tissue surface.

4. The needle penetrations should be at equal distances from the wound edge on both sides of the wound. The actual distance depends on the suture/ needle size but is generally agreed to be $2-3 \mathrm{~mm}$.

5. The passage of the needle through the tissues should follow the curvature of the needle.

6. When one side of the wound is fixed to the underlying tissues, one should first engage the mobile wound edge.

7. When one side of the wound is thicker than the other, the thinner wound edge should be engaged first.
8. When one side is deeper and the other more superficial, the deeper side should be engaged first.

9. The depth of needle penetration should be more than the distance from wound edge to needle penetration point. This will ensure eversion of wound edges.

10. The sutures should ensure proper tissue approximation of wound edges at the same time avoiding excessive tension on the tissues. Excessive tissue tension can lead to blanching, tear and necrosis. In case the edges are not approximating passively, the flaps may be undermined to achieve better mobilisation so that a tension-free closure can be achieved.

11. The knot should lie on one side of the wound, and not over the wound edges.

12. The spacing between the individual sutures depends on the type of tissue and the size of sutures. Generally placed 3-4 $\mathrm{mm}$ apart, they should be closer together at areas of underlying muscular activity and when the sutures are smaller in size.

\subsection{Specific Suturing techniques [3] (Video 11.1)}

There are quite a large number of techniques available to stitch a wound. Only the commonly used methods are mentioned.

\section{Factors to Be Considered on Specific Material and Technique}

1. Type of tissue,

2. Type of wound,

3. Time available,

4. Aesthetic requirements,

5. Functional needs,

6. Likely tension on the wounds.

7. Expected time for tissue healing.

\subsubsection{Simple Interrupted Suture}

A simple interrupted suture ('simple loop') is the most common method to suture a wound. This is the simplest design of wound closure. The suture passes once through each side of the wound in a simple loop and is then tied in a knot 
above the surface. Multiple such ties are made over the length of the wound, resulting in several independent sutures collectively securing the tissue edges together. The same technique is frequently employed for internal suturing of tissue layers also [4].

The needle penetrates the surface $2-3 \mathrm{mms}$ away from the wound edge on one side and proceeds into the subcutaneous tissue. The curve of the needle is then used to pass it through to the subcutaneous tissue on the opposite side. The needle then exits through the surface. The initial and final tags, now outside of the tissues, are then tied in a knot. The configuration inside the tissue is thus in the form of a loop. If the wound sides are of unequal depth, then the needle should travel deeper in the lower side while staying superficial on the higher side. This will help to correct the depth disparity and ensure proper surface levelling.

This technique is easy to learn and employ. This suture provides good tensile strength and carries minimal risk of wound oedema or impaired circulation. Several adjustments to the design are possible with this method depending on the wound characteristics. Since there is a series of multiple sutures, even if one suture fails, the others may provide sufficient strength to keep the wound edges together (Fig. 11.4).

The major drawback of this method of suturing is the high possibility of 'rail-road track' shaped scars caused due to the ingrowth of epithelium into the suture tracks. There is also a tendency to cause 'wound inversion' (depression of the surface at the wound site) due to tissue contracture during healing. The inversion can be prevented by making the suture configuration 'flask-shaped' inside the tissues, by making the needle travel farther laterally away from the wound within the tissues. When compared to continuous suturing techniques, the interrupted method is more time-consuming as it requires many more knots need to be tied.

\subsubsection{Simple Buried Suture}

The buried suture is a modification of the simple interrupted suture and is reserved for stitching the inner (deeper) tissue layers. The only difference is that the knot ends up in the tissues deeper to the suture loop, i.e. away from the surface. In essence, this is a simple interrupted suture in the reverse orientation.

The wound edge is first reflected using fine forceps or hooks. The needle is then inserted into the underside of the dermis on one side. The needle then proceeds along its curvature to exit in the wound edge more superficial to the initial bite. The needle then pierces the dermis wound edge (near the surface) on the opposite side, proceeds in a path mirroring its motion in the first side and comes out at a deeper point which corresponds to the first bite. Though the tie is done outside, the knot gets buried deep inside the tissues as it is tightened (Fig. 11.5).

This technique is extremely useful in suturing the inner tissue layers before the surface closure. As the knot is buried deep, it does not interfere with the closure of superficial layers. This aspect is extremely useful while closing cutaneous facial wounds.

Two major drawbacks have been observed with this suture. One is a skin dimpling which typically happens if the suture arc inadvertently involves the epidermis. Another problem is the tendency for wound inversion to occur. Small modifications such a set-back dermal buried suture or a vertical mattress buried suture can be employed to overcome this

Fig. 11.4 Simple interrupted suture technique

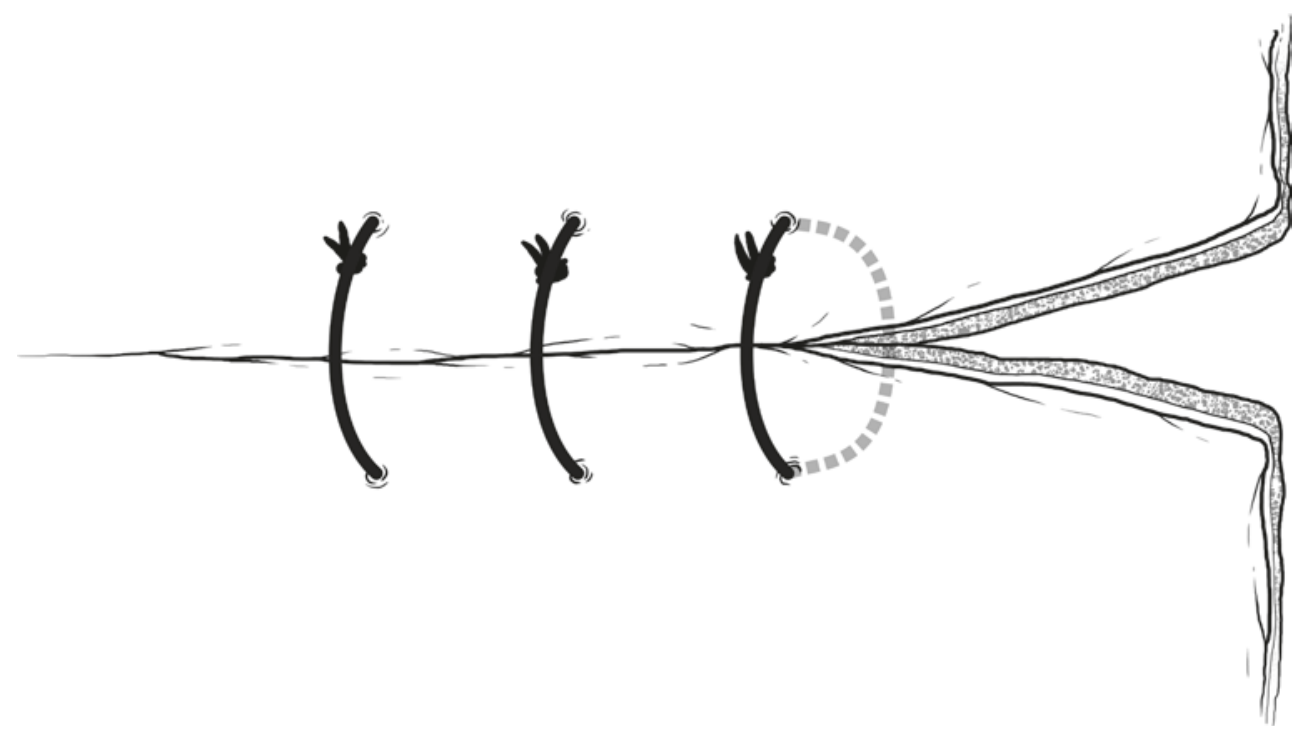


Fig. 11.5 Buried suture

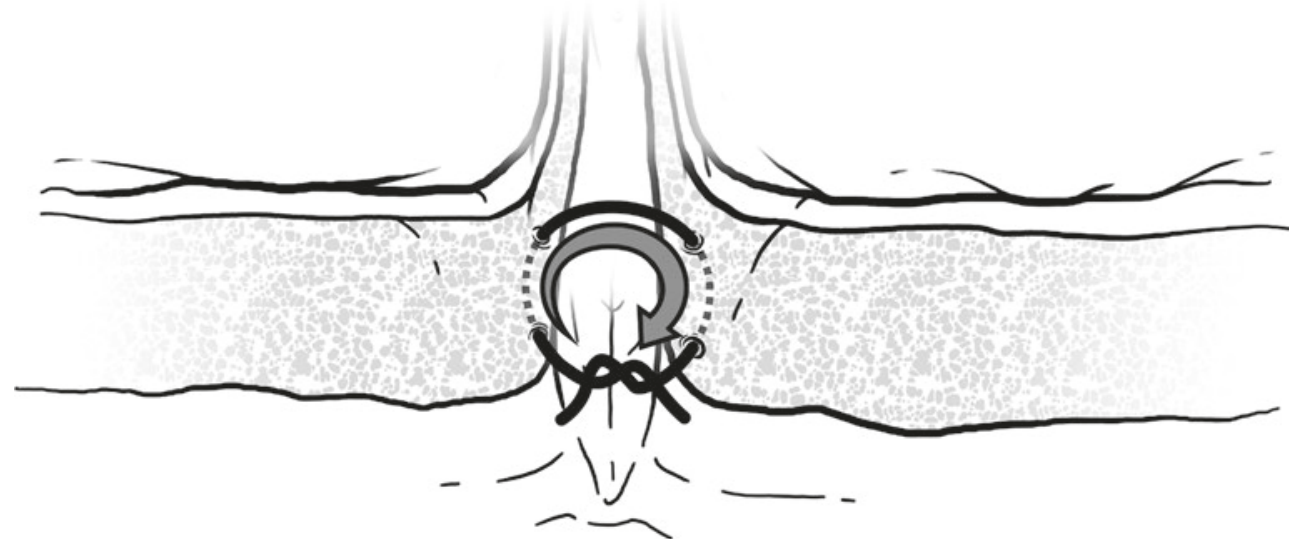

(C)Association of Oral and Maxillofacial Surgeons of India

Fig. 11.6 Vertical mattress suture

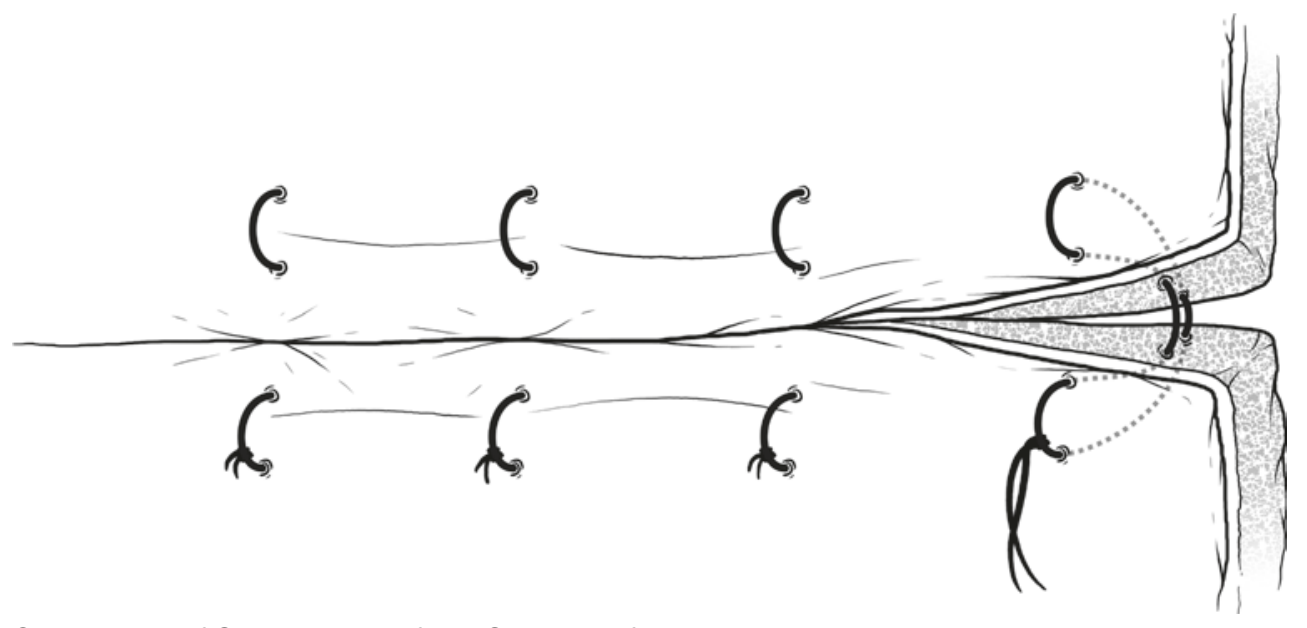

CAssociation of Oral and Maxillofacial Surgeons of India

problem. These modifications attempt to pass the bridging suture segments to be deeper, thus resulting in wound eversion.

\subsubsection{Vertical Mattress Suture}

The vertical mattress suture is in part similar to simple interrupted sutures but includes an additional suture bridge close to the wound edge. Also known as Donati Suture, or 'far-far, near-near' suture, this is the most frequently used suturing technique to obtain wound eversion [5]. This suture design is very popular in abdominal and limb surgeries.

The needle is first penetrated far from the wound edge (about $6 \mathrm{~mm}$ ), and then proceeds through the deeper tissue to the opposite side and emerges out at an equal distance from the edge. The needle is then reinserted at a point closer $(2-3 \mathrm{~mm})$ to the wound edge on the second side itself. It is then rotated superficially through the tissues and is exited on the other side at a corresponding near point. This results in a double bridging of the wound, one deep in the tissues and another superficial and nearer to the wound edge. Thus, both tags of the thread are now on one side of the wound. They are tied together gently (Fig. 11.6).

The primary advantage of this suture is the resultant wound eversion. This eversion is expected to compensate for the anticipated contracture occurring along the wound margin. As the suture is bridging the wound twice, the binding strength is more. Elimination of dead space is another obvious advantage. The suture thread does not pass the wound edge on the surface, minimising the chance of track marks.

On the flip side, fine wound edge approximation is almost never achieved with this suture. Excessive tightening could lead to over-constriction and sometimes exposure of the raw area. This may necessitate placement of additional interrupted sutures for better results.

The vertical mattress concept can be used in a buried suture situation also by ensuring that the more superficial suture thread passed back to the first side in a path parallel to the first, deeper suture bridge. This will result in a better wound eversion. 


\subsubsection{Simple Continuous Suture}

Simple continuous suture ('running loop', 'standard running suture') is a good method for rapid closure of small wounds. It gives an even distribution of tension all along the wound span. It combines many benefits of the simple interrupted sutures with the additional advantage of a quicker finish.

The technique is very simple. The first part follows the same process as a simple interrupted suture. The first knot becomes the anchoring knot for the ensuing running line. The tags are not cut and the longer tag (with the needle) is used for making the remaining loops. The needle is inserted back into the tissues a few millimetres away from the first piercing. Then the needle (followed by the thread) passes through the tissues in a path parallel to the first loop. Once it comes out of the second loop, it is not tied. Instead, the loop is tightened and the thread crosses over obliquely across the wound and enters the tissue surface again, a few millimetres away from the second piercing. This process continues over and over until one reaches the other end of the wound. As the last loop goes through the tissues, the thread is only partially pulled through, leaving some loose thread on the opposite side. The suture is then tied to this loose thread for the final knot. Thus, the suture material runs across the wound in repetitive loops, and there are only two knots-one at either end (Fig. 11.7).

The obvious advantages of this technique are the quickness of suture placement and the ease of avoiding many knots. Also, if the tissue wells up in one site, the remaining part of the suture can provide some compensatory slack.

The principal drawback is that the integrity of the entire suture line is vested in just two knots. Any breakage to the suture at any point leads to the entire line getting untied. Since the loops are in continuous succession, fine-tuning of design for each loop is not possible. Also, since the tension is the same across all loops, the areas of the wound with greater tension, usually the central part, may tend to gape.

\subsubsection{Locking Continuous Suture}

This is a variation of the simple continuous suture technique used for surface wounds. Each loop of the continuous suture is 'locked' on itself before making the next loop. This is the most popular continuous suturing technique especially for closing wounds over long spans. As with other superficial continuous sutures, this method is frequently used as a surface layer after the internal tissue layers have been closed.

Similar to the simple continuous technique, the first loop is passed and the knot tied. After the second loop has gone through the tissues and exited, the suture is not immediately tightened. The needle and the leading thread it is made to pass through the earlier loop. After making this 'lock', the suture is tightened and is then passed into the tissues for the third loop. The assistant should maintain this tension until the next loop is passed. This process is repeated for the entire line of succeeding loops (Fig. 11.8).

The locking helps to align the tissues in a proper anatomic orientation perpendicular to the wound. There is an added haemostatic effect due to the tension on tissues. Uniform degree of tension is maintained across all the loops. At the same time, the running locks partially detach the tension on the individual loops from one another. Thus, to an extent, individual control of tension depending on the site can be obtained.

This technique inherits the main disadvantage of the simple continuous sutures of being dependent on only two knots, and the risk of complete loss of suture integrity in case of breakage at any point. The locks, if they are too tight, may cause vascular compromise of underlying tissues.

Fig. 11.7 Simple continuous suture

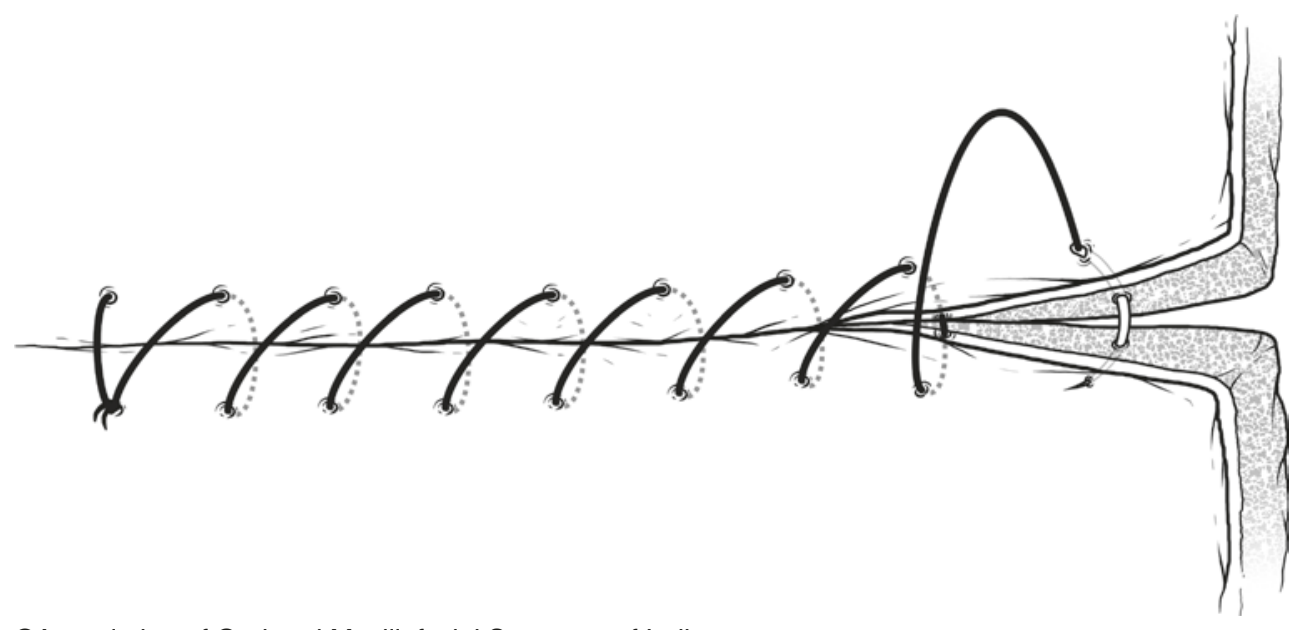


Fig. 11.8 Locking

continuous suture

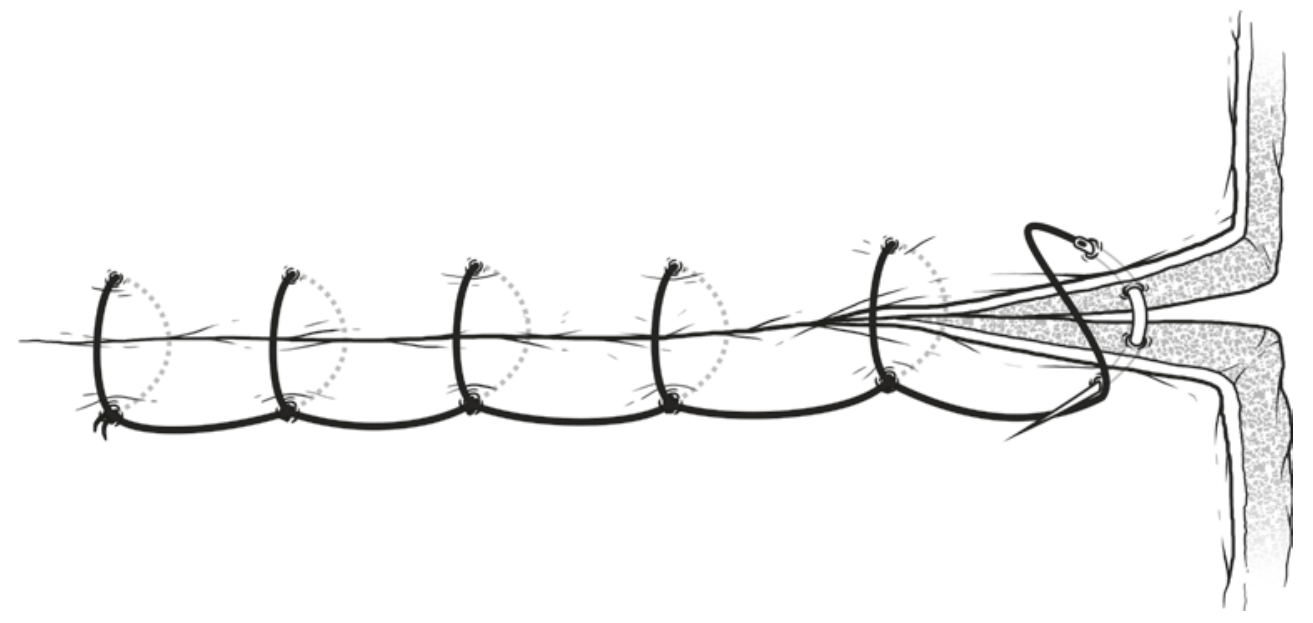

CAssociation of Oral and Maxillofacial Surgeons of India

Fig. 11.9 Subcuticular suture

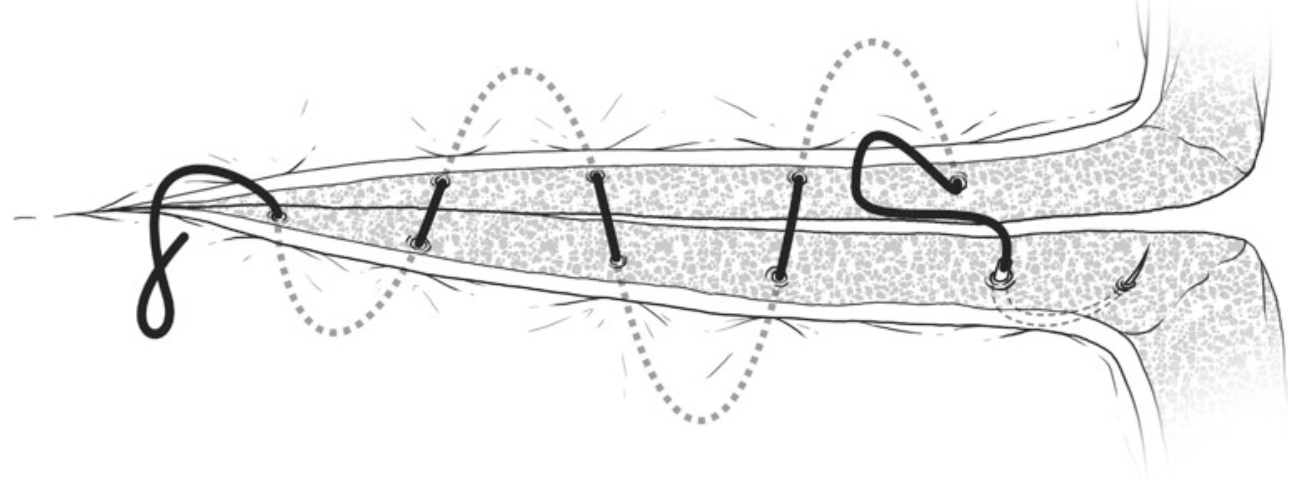

@Association of Oral and Maxillofacial Surgeons of India

\subsubsection{Subcuticular Suture}

This is a mostly buried, continuous, epidermal wound closure which is used for surface closure. The suture ends exit a few mms away from the wound corner. The subcuticular suture is employed only after the deeper structures and dermis have been secured well with absorbable sutures. It is popular as an aesthetic suture for face [6].

The suture may be absorbable or non-absorbable, and is usually thin (size 5-0 or 6-0). The needle insertion is at one end of the wound, $2-5 \mathrm{~mm}$ away from the apex. It is passed along the curve into the wound, where it exits in the interior, close to the apex. Then the needle is inserted again into the dermis on any one side of the wound edge walls. Thereafter, it passes horizontally parallel to the surface, and following the needle curve to come out into the wound interior a small distance away. The same step is then repeated on the other side of the wound. This process is repeated till one reaches the other end of the wound, wherein the needle is made to pierce the far end apex and to come out in the surface. This last step is a mirror image of the initial steps. Then each of the suture tags on both sides is tied separately on to itself. Alternatively, the tags may be secured with adhesive strips, surgical tape or tissue glue (Figs. 11.9 and 11.10).

The biggest advantage of this technique is the much decreased risk of scars. The close approximation achieved in the dermis region makes the need for a further surface suturing unnecessary. The tension is aligned centrally across the wound and is evenly distributed all along the length of the suture. Also, this technique is highly suitable in cases where the suture material is required to stay in place for a long period of time.

On the negative side, the subcuticular suture takes longer time to perform. Leaving a large quantity of foreign material in situ can increase the risk of foreign body reaction and infection. If non-absorbable material is used, there is a minor risk of long and thin suture track following removal. If absorbable material is used, an undyed suture should be cho- 
sen to prevent cutaneous visibility. Incorrect technique can leave small segments of exposed raw area, which need to be addressed with additional surface sutures.

\subsubsection{Purse-String Suture}

This is essentially a modification of the simple continuous suture, and is designed to reduce the size of a two-dimensional surface defect. It is not a cosmetically superior technique and is rarely used in the face. The purse-string effect causes a puckering in the surrounding skin tissue. But this is an effective method to reduce wound area. It may also be used to achieve haemostasis.

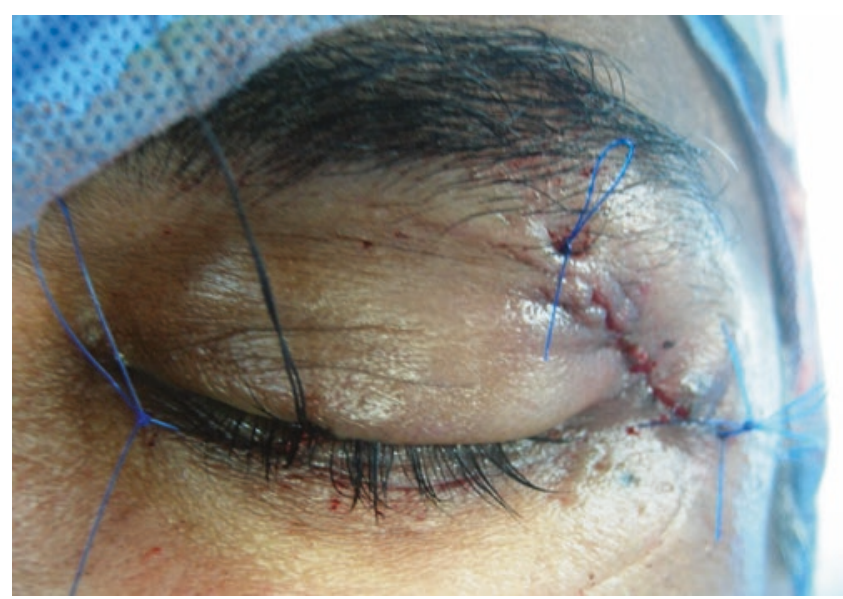

CAssociation of Oral and Maxillofacial Surgeons of India

Fig. 11.10 Subcuticular suture at the subciliary incision site and at the pre-existing lateral orbital laceration in a case of zygomatic complex fracture fixation
At first, the wound edges of the defect need to be freshened and underlined. The needle and thread is passed along the edge of the defect in a course running parallel to the wound edge. Thereafter, it continues in a series of loops running along the edge of the surface wound along its entire circumference. As the thread completes the full distance and reaches near the initial needle entry, it is pulled taut, leading to complete or partial closure of the wound. Then the tags are tied together.

\subsubsection{Three-Point Suture}

Also known as 'tip stitch' or 'half-buried horizontal mattress suture', this technique is used for managing a situation where three ends of the tissue have to be sutured together. In maxillofacial surgery, this situation is encountered while repairing V-shaped lacerations and while closing flaps with sharp corners (such as the triangular flap at the vermilion border for cleft lip repair)

This suture is placed only after the flaps are brought into position using buried dermal sutures. The surface suture thread is used, that is, 6-0 for face and 3-0 for scalp. The needle is first inserted into one side of the non-flap side of the wound. It follows the needle curve to exit in the inner aspect of the wound. The next tissue insertion is into the superficial dermis of the flap tip. Then the needle passes horizontally and comes out through the dermis on the other side of the flap tip. After releasing from the flap tip, it re-enters the dermis of the non-flap side on the other side and comes out through the skin at a point corresponding to the initial entry. The tags are then knotted together (Figs. 11.11 and 11.12).

Fig. 11.11 Three-point suture technique

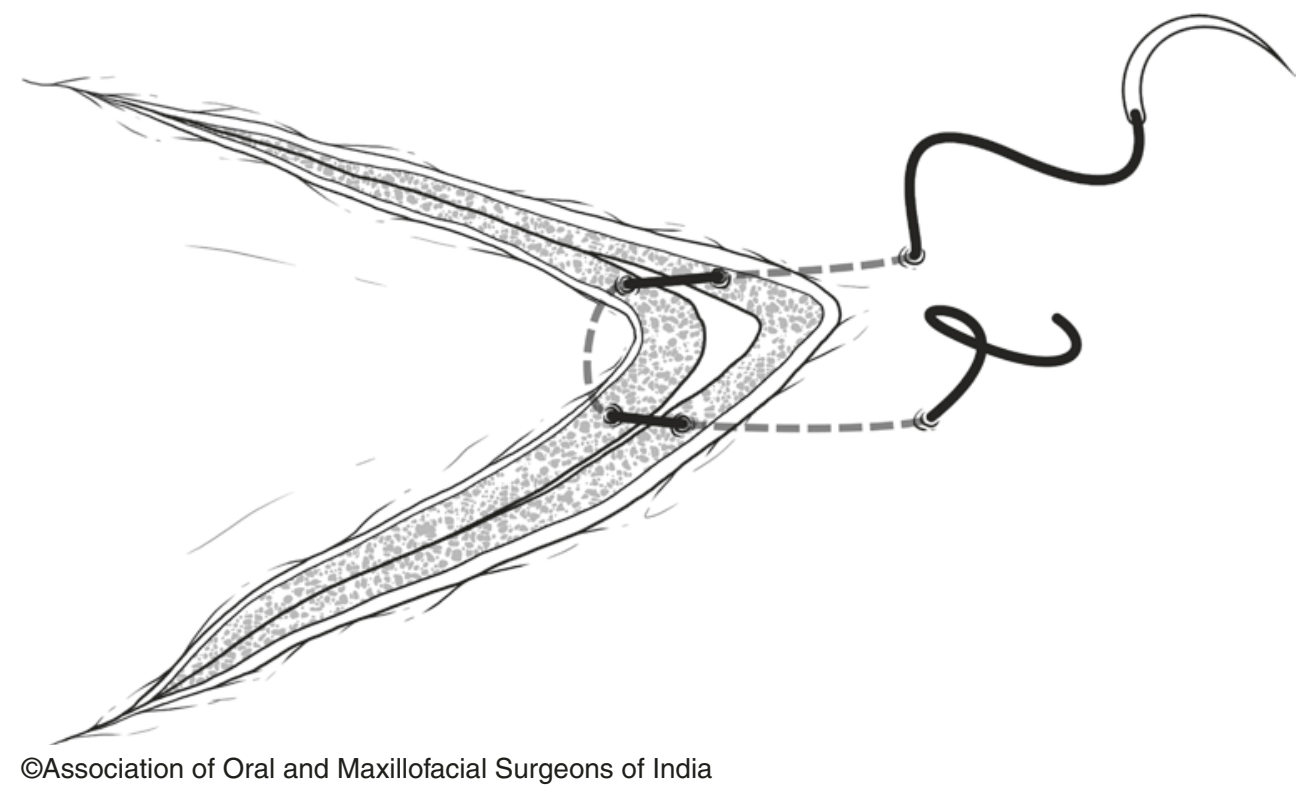




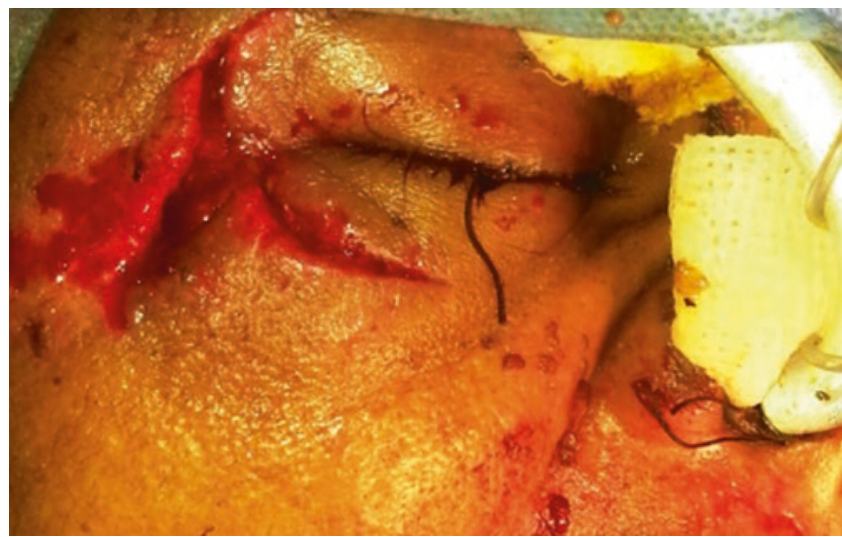

CAssociation of Oral and Maxillofacial Surgeons of India

Fig. 11.12 A laceration that needs three-point suturing

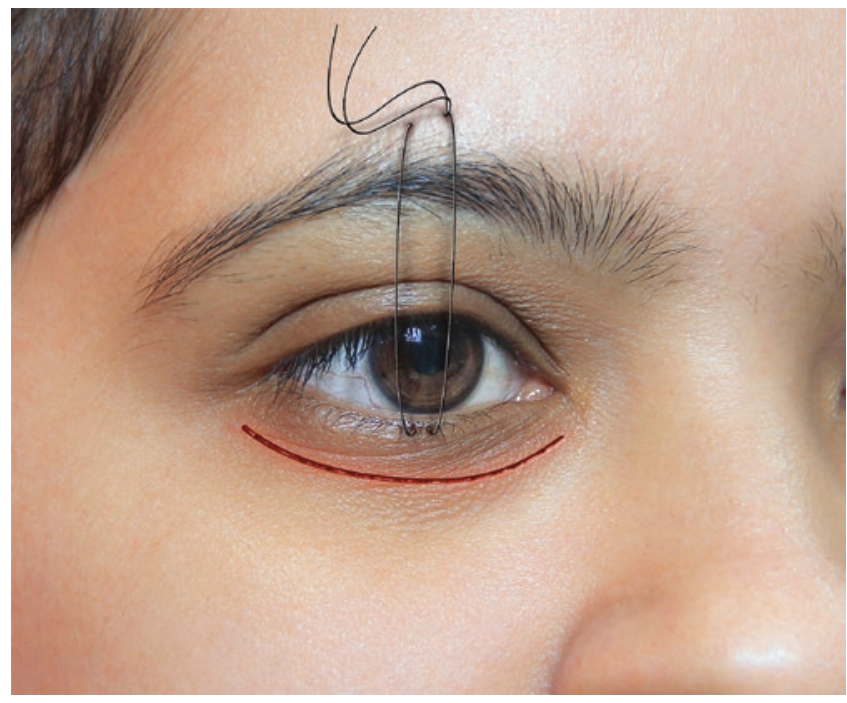

CAssociation of Oral and Maxillofacial Surgeons of India

Fig. 11.13 Frost suture

\subsubsection{Frost Suture}

The Frost suture is a temporary eyelid suspension suture, used to ensure proper lower eyelid placement during the post-operative period [7].

After the closure of the lower eyelid incision, a needle bite is taken on the tarsal plate or just inferior to it. Then a second bite is taken just above the eyebrow, ensuring the anatomic position and alignment of the lower lid. These tags are then tied or secured with tapes or tissue glue (Fig. 11.13).

These sutures may be removed on third post-operative day, but need to be kept longer if there has been significant trauma to the tissues.

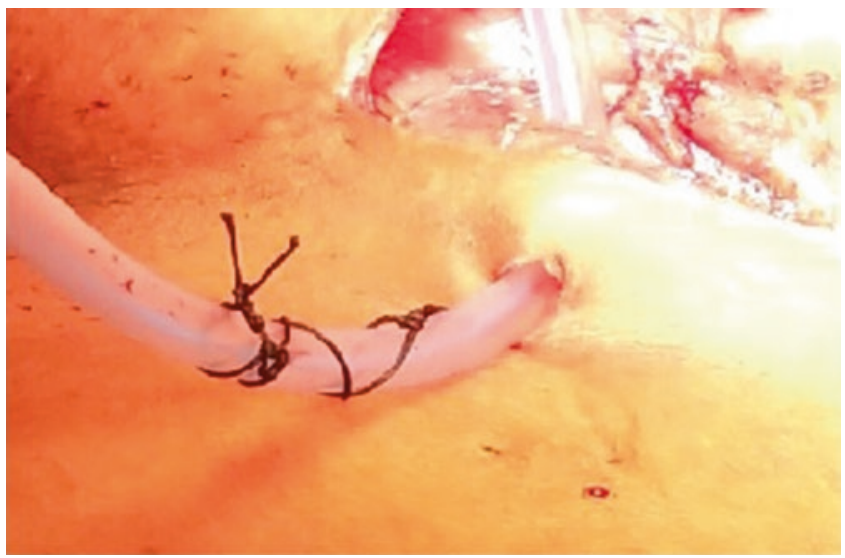

CAssociation of Oral and Maxillofacial Surgeons of India

Fig. 11.14 Drain anchoring suture

\subsubsection{Drain Anchoring Suture}

Different types of drains are used in surgery, such as corrugated rubber drain, the suction drain, intercostal drain, etc. After the drain has been inserted, it needs to be securely fixed to the body to prevent displacement.

The most common means to secure drains is the Roman Garter method, which uses silk sutures. A strong bite is first taken on the skin near the drain entry site. After making a knot, the two suture tags go around the drain tube in a series of windings. A knot may be tied after each 2 or 3 turns around the tube. The large number of windings around the tube increases the friction, holding the tube in position without dislodgement (Fig. 11.14).

Other techniques which have been described include the use of nylon suture, safety pin, drain clip, adhesives and Tie-lok.

\subsection{Dentoalveolar Suturing}

The tissues and the surgical environment in the dentoalveolar region are different from other parts of the body. Consequently, the wound closure requirements also differ. Wound closure in dentoalveolar surgery involves suturing in situations such as

1. Suturing of buccal and/or lingual flaps back to its place after dentoalveolar surgery around teeth or implant accessories.

2. Wound closure after tooth extraction.

3. Flap closure in edentulous ridge. 
In most cases, the needle passage is through keratinised mucosa with little or no subcutaneous tissue, obviating the need for multilayered closure. The presence of teeth or artificial crown on one side of the flap forces the surgeon to innovate on the basic suture design. Another difference is when the extraction wound is closed-the wound is usually closed only at the edges and is largely left open in the middle. Also, the suture site will have to endure itself in a challenging environment with the presence of saliva, food materials and a very dynamic milieu where tongue movements and masticatory forces abound [8].

If marginal gingiva is not involved in the flap (as in a semilunar flap), the wound closure models are usually not different from the general cutaneous designs which were discussed in the previous section.

\subsubsection{Simple Interrupted Suture (Interdental Suture)}

As in any case of wound closure, the simple interrupted suture is the mainstay in closing dentoalveolar flaps [9]. In general, it involves suturing the detached interdental papillae together. The needle passes from the buccal aspect of the buccal flap, emerges on the inside of the flap and then passes between the tooth roots to enter the inner aspect of the lin-

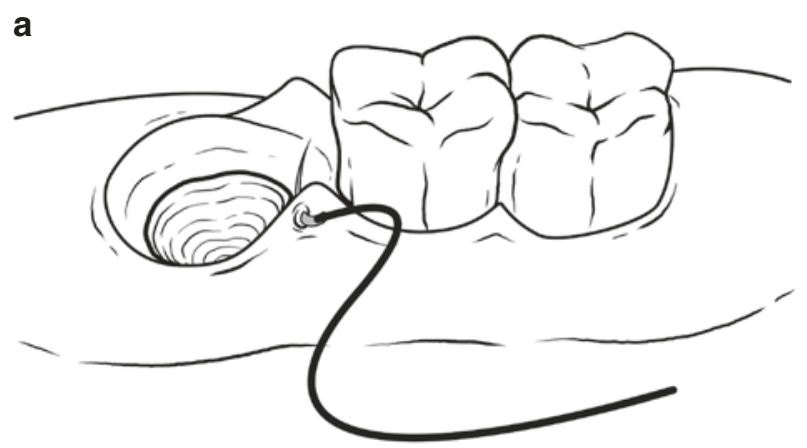

C

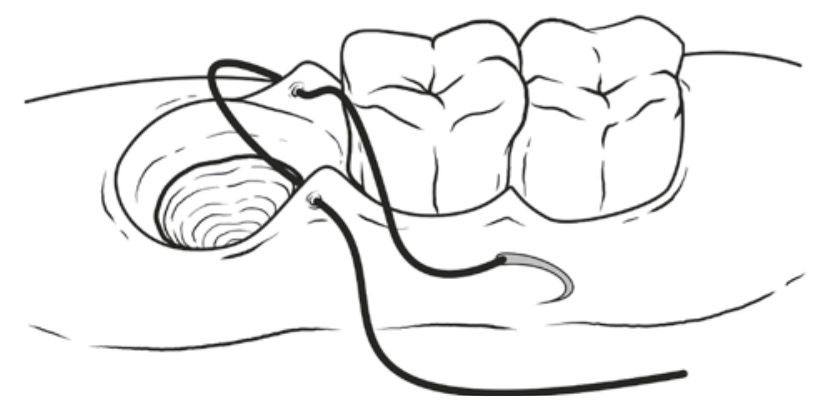

CAssociation of Oral and Maxillofacial Surgeons of India gual flap. As it pierces the lingual flap and emerges through the mucosa, the needle is turned back and is taken back via the interdental region to the buccal side. The two suture tags are then tied together. Thus, the knot remains on the buccal side and is accessible for removal.

If only one flap (usually buccal) has been raised, the needle can still pass through the attached lingual flap and the suture design stays the same. In post-extraction wounds also, the procedure is the same, and the tooth socket space is not totally closed.

\subsubsection{Interrupted Reversing Suture}

In this modified interrupted suture, the direction of the needle is reversed for engaging the lingual papilla. Once it passes through the buccal papilla and reaches the lingual side, the needle orientation is reversed and it is made to enter the lingual papilla from outside (lingual side). The needle exits the flap on the inside, passed across to the buccal side and is then tied. Thus, both the papillae are engaged in an 'outside-to-inside' orientation, ending in a figure-of-eight formation in the vertical plane (Fig. 11.15).

This technique is especially useful in cases where both buccal and lingual flaps are raised during surgery, such as in periodontal surgeries.

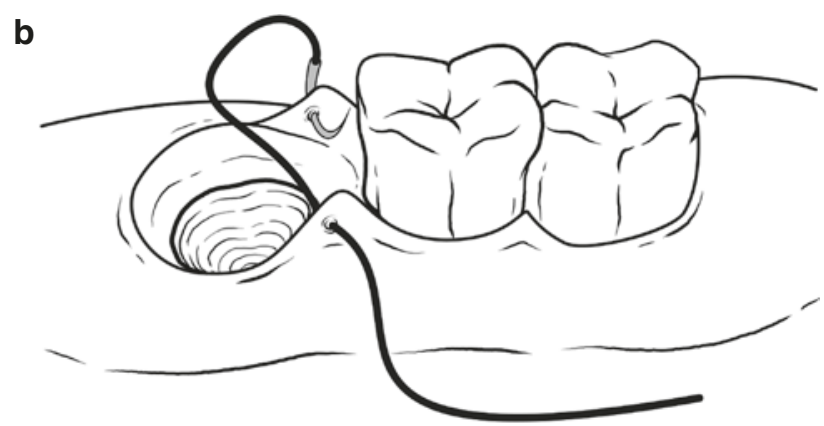

d

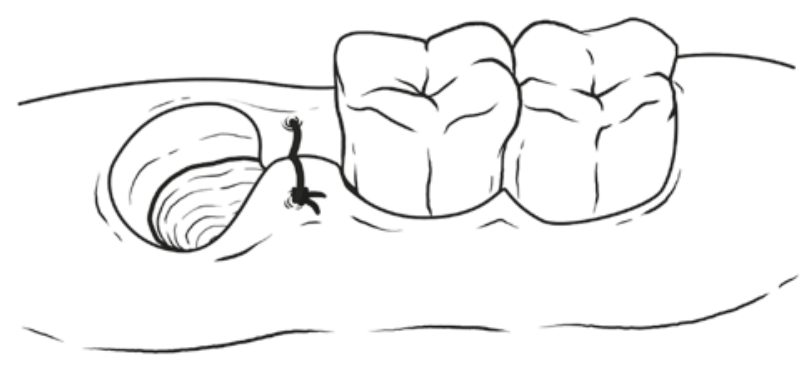

Fig. 11.15 Interrupted reversing interdental suture 


\subsubsection{Vertical Mattress Suture}

Vertical mattress technique in dentoalveolar surgery is a modification of the papilla-attaching simple interrupted technique. After the initial passage of the needle through the buccal and lingual papillae, the needle is turned back and picks a small bite at the tip of the lingual papilla before passing back to the buccal side. Then it again pierces the tip of buccal papilla before knot-tying.

While this technique helps in pressing the papilla into the interdental space, it is not a popular technique because of the difficulty in getting a solid bite at the papilla tip without 'cutting through'. Obviously, one needs to use an atraumatic needle with a small thread (4-0 or smaller) for this purpose.

\subsubsection{Horizontal Mattress Suture}

This is a two-dimensional suturing technique where the suture thread is spread in a horizontal fashion in the tissues. It is not very popular for general cutaneous suturing but is especially useful in dentoalveolar surgery.

When closing flaps in edentulous areas, the needle first passes through both flaps. Then it is reinserted into the flap on the same side, a little distance away from the earlier exit point. The reinserted needle now passes through both flaps and emerges out a similar distance away from the initial entry point. Both the thread tags, now on the same side, are tied together (Fig. 11.16).

In dentulous areas, this technique sutures two adjacent papillae together, eventually tying four papillae segments around a tooth in a horizontal square fashion. To close extraction wounds, only the papillae are approximated and the tooth socket space is not closed completely.

The advantage of this technique is that a single horizontal mattress suture serves like two separate interrupted sutures, thus helping to reduce the number of sutures. It compresses the wound from four corners and helps in haemostasis. It also results in some degree of wound eversion.

\subsubsection{Horizontal Mattress Modification: Dental Anchor Suture}

In this modification, the suture does not pass through the lingual soft tissues. After passing through the buccal papilla, the suture passes through the interdental region into the lingual side. Without engaging the lingual papilla, it goes around the tooth and comes back to the buccal side via the interdental space on the other side. Then it engages the buccal papilla before being tied. In this way, it secures the papilla to the bone using the tooth as its 'anchor'.

This technique can also be used in a reversing fashion, where only the papilla on the lingual side is engaged and the buccal side papillae are not pierced.

\subsubsection{Horizontal Mattress Modification: Mattress Sling Suture}

This technique attempts to combine the benefits of vertical mattress suture with those of horizontal mattress suture. The basic technique goes similar to the horizontal mattress but the final tag re-enters the buccal papilla at the tip and traverses a reverse path around the tooth to exit near the initial entry. In this way, it ensures the engagement of both buccal papillae in a vertical mattress fashion.

\subsubsection{Horizontal Mattress Modification: Figure-of-Eight Suture (Cruciate Mattress Suture/Cross Suture)}

The figure-of-eight suture is the most popular modification of the horizontal mattress technique. This is used mostly in closure of extraction wounds, and never in the presence of teeth at the wound site.

The needle first penetrates the buccal papilla on one side and then the lingual papilla. Then the thread crosses across the edentulous site, and the needle is reversed in orientation to pierce the buccal papilla of the other side from outside
Fig. 11.16 Horizontal mattress suture (extraction wound)

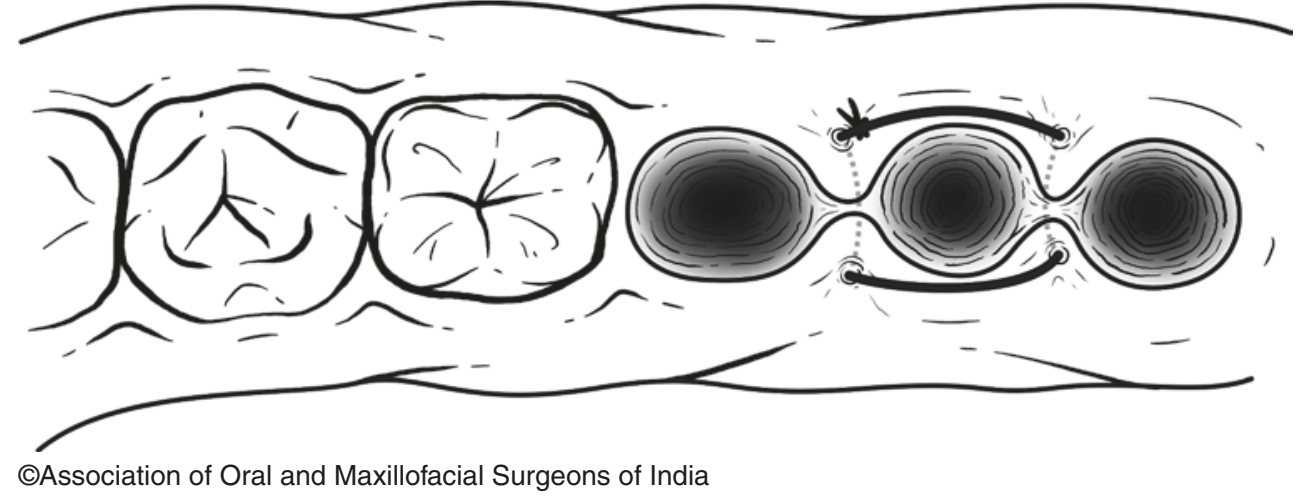


Fig. 11.17 Figure-of-eight suture

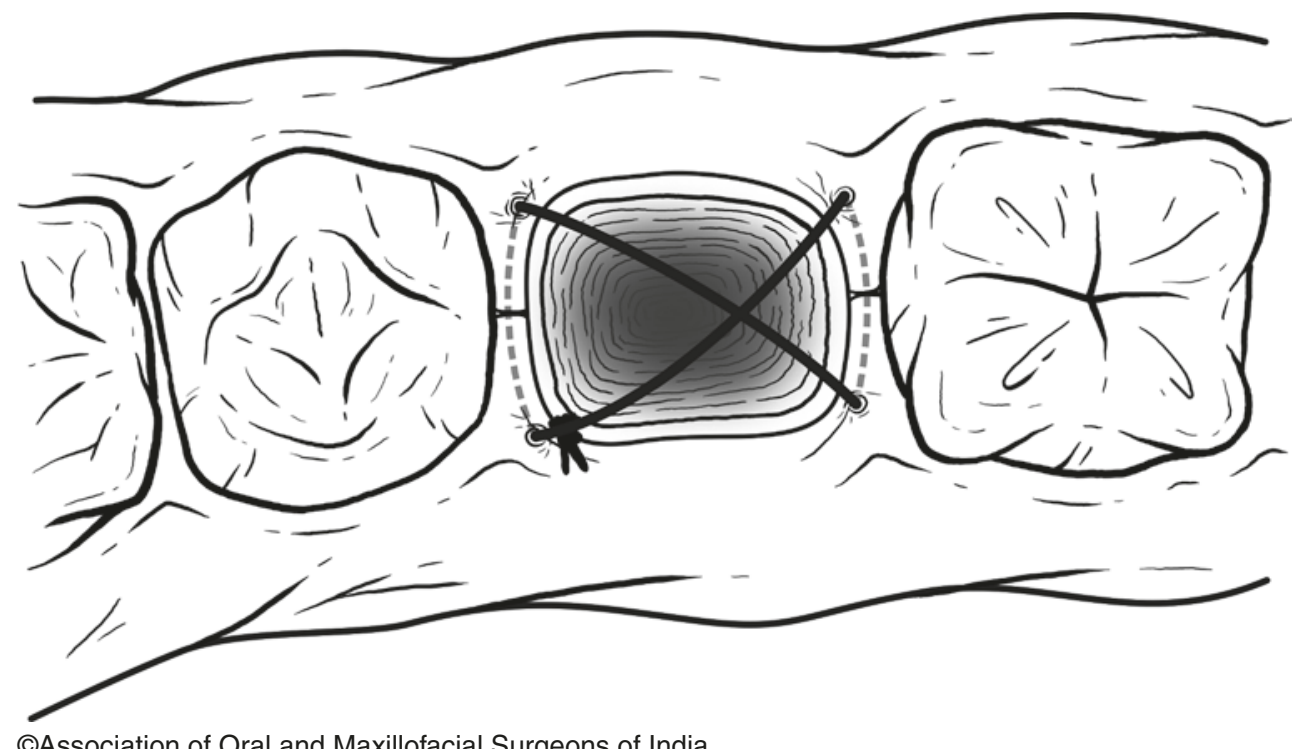

(C)Association of Oral and Maxillofacial Surgeons of India (buccal side) to pass through the lingual papilla and exit on lingual side. Then the thread passes across the site to be tied with the initial tag. The suture thus ends with the appearance of suture material crossing the wound site in a 'cross' shape (Fig. 11.17).

Many surgeons consider the figure-of-eight suture to be the most comprehensive way to suture a single tooth extraction site. This method ensures an even tension on the tissues from four corners, effectively constricting the wound. There is a positive effect on haemostasis also, due to the tension on the flap and due to its presence a mechanical barrier to clot loss.

\subsubsection{Simple Continuous Suture}

The continuous sutures are almost exclusively used for edentulous situations including post-extraction closure. After making a conventional interrupted suture knot, the longer tag is not cut and is instead used to make a series of 'running loops' approximating the succeeding pair of papillae one after the other. Gentle tension must be maintained on the thread to keep the loops tight while the needle passes through the next tissue. Knots are not tied for each loop but is done only at the other end of the long wound. Some surgeons prefer to tie a knot after 3 or 4 running loops. In the end, the part of the suture passing through the inner tissues are perpendicular to the wound, and the superficial, exposed parts lie across the wound in an oblique fashion.

The continuous sutures save time and effort while closing wounds of long span. It also ensures an even distribution of tension across the wound. The obvious disadvantage is the fact that if one loop or part of the suture is compromised (by

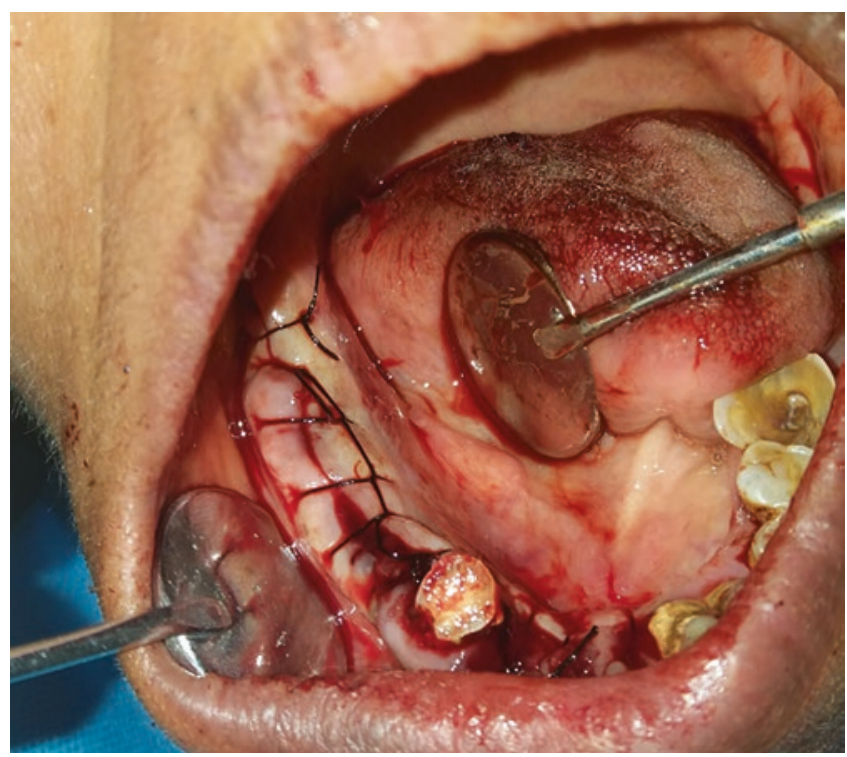

CAssociation of Oral and Maxillofacial Surgeons of India

Fig. 11.18 Continuous locking suture (dentoalveolar)

untying, cutting through or loosening), the entire suture line gets loosened.

\subsubsection{Continuous Locking Suture}

The continuous locking suture is a continuous suture in which a 'lock' is incorporated by passing the thread under the previous loop before it is pulled through the tissue. It is important to keep maintaining the tension on the previous loops as the needle makes the next pass through the tissue (Fig. 11.18). 
This technique ensures a better orientation of the sutures with respect to the wound. The superficial, exposed parts of the suture are oriented perpendicular to the wound, leading to better anatomic wound approximation.

\subsection{Suture Removal}

Non-absorbable sutures on skin and mucosa should be removed after the wound surface has achieved initial stability. The timing of suture removal is very important. They should remain in tissue long enough to prevent dehiscence and scar spread. On the other hand, early removal reduces tissue reaction and suture marks.

Sutures on facial skin and in oral cavity are usually removed in 5-7 days. The recommended interval is 3-5 days for eyelids, 7 days for neck and 7-10 days for scalp. Those on trunk and limbs should remain in place for 10-14 days.

The suture line is cleansed with antiseptic. The knot is grasped and is pulled away mildly from the surface and to one side of the wound. An uncontaminated segment of the thread is exposed on the other side by this pull. The thread is cut at this segment near to the surface. Then the suture is pulled out, making sure that no contaminated (exposed) part is ragged through the tissues.

In the case of a continuous suture, every single loop should be cut and pulled out separately. A subcuticular suture is removed by cutting the knot off at any one end, and then pulling the suture out gently from the other end. It is important to make sure that the suture does not break within the tissues.

\subsection{Other Wound Closure Methods}

\subsubsection{Staples}

The use of specialised staples for wound closure was popularized in 1900s by the Hungarian surgeon Hümér Hültl, known as the 'father of surgical stapling'. Compared to suturing, surgical stapling is a quicker method to close the skin in large wounds, and the inflammatory response is relatively less. Staples provide good wound edge eversion without strangulation of the tissue. It is an excellent method to employ in cases which require quick wound closure and where aesthesis is not a major concern [10] (Fig. 11.19).

Though titanium staples were initially used, almost all of the contemporary hardware is stainless steel. However, titanium retains the advantages of being biocompatible and MRI-compatible. Bioresorbable staples, based on polyglycolic acid, are also available. The stapler devise itself may be of stainless steel (reusable) or plastic (disposable), into which the disposable staple cartridges can be loaded.

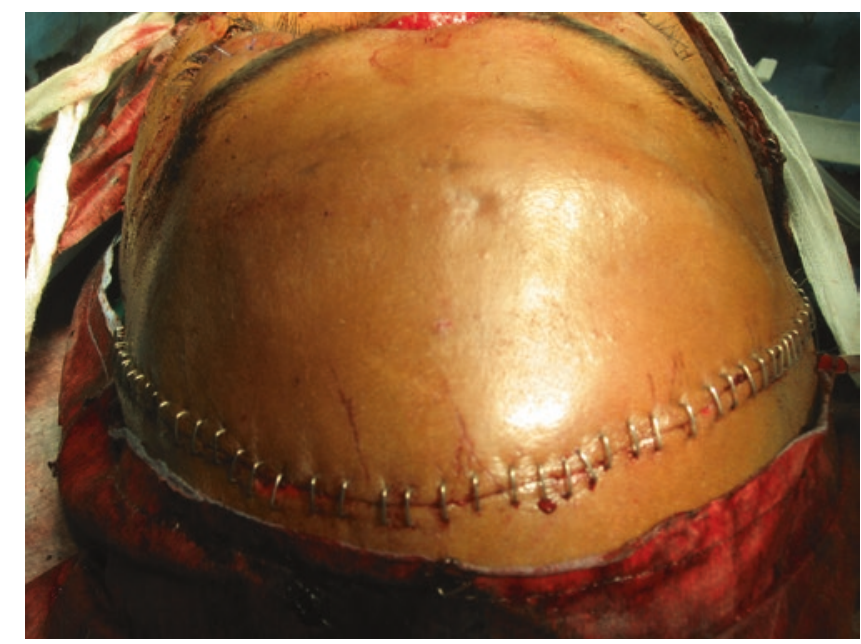

CAssociation of Oral and Maxillofacial Surgeons of India

Fig. 11.19 Stapled scalp incision in a coronal approach

In maxillofacial surgery, staples are frequently used to close scalp wounds and neck incisions, following the closure of internal layers with conventional sutures. Staples are also popular in reconstructive surgery to secure skin grafts and to close the flap donor sites. They are not generally used to close facial wounds, since there is a tendency to produce 'rail-road track' scars. Skin staples are removed after 7-10 days with a specialized staple remover device.

\subsubsection{Tapes}

Different types of surgical adhesive tapes are used to effect wound closure, to reduce tension on sutured wounds and to reinforce wound site after suture removal.

The indications of this method for wound closure per se are limited. Tapes can be used to close superficial lacerations where tissue tension is minimal. They are also employed in the closure of superficial layer after buried dermal sutures have been employed for wound edge approximation and tension reduction. A major indication is its use an additional reinforcement and protection over sutured wounds. The advantages of using tapes for wound closure are.

(a) They are rapid and easy to perform.

(b) The tape application is painless (patient anxiety and discomfort are minimal).

(c) Residual suture track scars are avoided.

(d) There is no need for a review visit to remove the tape.

(e) Tapes, being non-invasive, are less prone to infection than other methods.

(f) They are suitable for thin, fragile skin of the elderly and infants. 
On the other hand, there are significant limitations to the use of tapes to close wounds. The most obvious contraindication is an area under significant tension. Tapes are difficult to apply on to highly convex surfaces, irregular wounds and in areas of tissue laxity. They do not attach well to wet surfaces (e.g. oral mucosa). They may get detached easily in hairy areas and those which tend to sweat.

These tapes are typically made of synthetic reinforced material with a hypoallergenic adhesive and are made porous to make them 'breathable' for skin. Some products are made elastic to account for oedema tension while some have incorporated antibiotic to reduce the incidence of surgical site infection. Modifications of tape-based wound closure include incorporation of a zip-lock mechanism and a clip attachment, both techniques designed to pull the wound edges together for better approximation.

\subsubsection{Adhesives}

The use of tissue adhesives is an efficient way to close wounds in select cases. Just like surgical tapes, tissue adhesives can be used to approximate wounds that do not require deep-layer closure and do not have significant tension on the edges [11].

All currently available tissue adhesives are chemically cyanoacrylates (esters of cyanoacrylic acid). These compounds were discovered by Ardis in 1949 and were first used in surgery by Coover in 1959. These are chemically similar to methacrylates, the only difference being the methyl $\left(\mathrm{CH}_{3}\right)$ group substituted with cyano/nitrile $(\mathrm{CN})$ group. The water present on the skin or mucosal surface activates the acryl groups in the resin to rapidly polymerise and form long, strong chains. Three types of tissue adhesives are currently available for clinical use-2-octyl cyanoacrylate, N-butyl-2-cyanoacrylate and isoamyl 2-cyanoacrylate. The adhesion involves two mechanisms-mechanical interlocking into the surface irregularities and chemical covalent bonding with the nucleophilic amine groups on skin surfaces.

Tissue adhesives are mainly used to treat superficial skin tears (that do not extend past the dermis) and for surface closure after the deeper layers are secured with sutures. Other indications include stabilisation of bone fragments during plating, sealing of CSF leaks and sinus perforations, attaching grafts, achieving peripheral nerve reanastomosis and closure of dentoalveolar flaps [12]. They are also widely used to achieve haemostasis at surgical sites and as a biologic cover on ulcers. The relative contraindications include wounds over or near joints and wounds under significant static or dynamic skin tension. Also animal bite wounds, crushed wounds and wounds in high friction areas and those with cross mucocutaneous borders are not considered suitable for a closure using adhesives.

The procedure for using the tissue adhesives for surface closure involves thorough cleaning and haemostasis. Though moisture is a prerequisite for adhesion, the presence of excessive water or blood at the site is detrimental to a good result. The surface is dried before applying the adhesive in at least three to four thin layers along the length of the wound's surface. It is advisable to extend it approximately 5-10 mm from each side of the wound. The edges of the wound are held together for at least 1 minute as the adhesive dries.

The main advantages of tissue adhesives over other wound closure methods are the rapidity and painless application [13]. In addition, the suture track scars are avoided, leading to a much better cosmetic result. The risk of suture site infection is also found to be less. The material sloughs off in 5-10 days, as the skin sheds.

The obvious disadvantage is that it cannot be used in areas of tension. Additionally, there is a minor risk of toxicity and foreign body reactions. If the clinician's gloved fingers, gauze or plastic instruments contact the tissue adhesive during application, these materials may adhere to the patient's skin.

\subsection{Conclusion}

Wound care primarily involves measures to ensure that the wound heals quickly without going through adverse situations such as infections. Along with mechanical debridement, antibiotic medication and dressings, the various wound closure methods form the foundation of wound care practices. Depending on the patient needs and the wound types, one should choose from a wide variety of materials and techniques to implement effective wound closure.

\section{References}

1. Buckley MJ, Keller JC. Emerging biomaterials and tissue engineering. Oral Maxillofac Surg Clin North Am. 2002 Feb;14(1):ix.

2. Herford AS, Haug RH. Emerging biomaterials and techniques in tissue regeneration. Oral Maxillofac Surg Clin N Am. 2017;29(1):1-120.

3. Kandor J. Atlas of suturing techniques. Approaches to surgical wound, laceration, and cosmetic repair. New York: McGraw-Hill; 2016.

4. Hupp JR. Guide to suturing. Section 2. J Oral Maxfac Surg. Aug 2015;73(Suppl 1):9-35.

5. Kudur MH, Pai SB, Sripathi H, Prabhu S. Sutures and suturing techniques in skin closure. Indian J Dermatol Venereol Leprol. 2009;75(4):425-34.

6. Gusman DN. Suture materials and techniques. In: Lower extremity soft tissue and cutaneous plastic surgery, vol. 1. Nottingham: Saunders; 2012. p. 77-100. 
7. Connolly KL, Albertini JG, Miller CJ, Ozog DM. The suspension (frost) suture: experience and applications. Dermatol Surg. 2015 Mar;41(3):406-10.

8. Koshak HH. Dental suturing materials and techniques. Glob J Otolaryngol. 2017;12(2):1-11. GJO.MS.ID.555833

9. Griffin TJ, Hur Y, Bu J. Basic suture techniques for oral mucosa. Clin Adv Periodontics. 2011, Nov;1(3):221-32.

10. Iavazzo C, Gkegkes ID, Vouloumanou EK, Mamais I, Peppas G, Fallagas ME. Sutures versus staples for the management of surgical wounds: a meta-analysis of randomized controlled trials. Am Surg. Sept 2011;77(9):1206-21.
11. Tuptis A, Salma I. Fibrin sealant in maxillofacial surgery (literature review). https://www.rsu.lv/en/scientific-papers/fibrin-sealantmaxillofacial-surgery-literature-review. 2015.

12. Kaderi MA, Menaka KB, Metgud RM, Gharat MR, Naik PS, Ajmani JM, et al. In-vitro evaluation of antibacterial potential of cyanoacrylate tissue for intra-oral wound closure. J Dent Mater Tech. Sep 2017;6(4):163-9.

13. Oladega AA, James O, Adeyemo WL. Cyanoacrylate tissue adhesive or silk suture for closure of surgical wound following removal of an impacted mandibular third molar: a randomized controlled study. J Craniomaxillofac Surg. 2019 Jan;47(1):93-98.

Open Access This chapter is licensed under the terms of the Creative Commons Attribution 4.0 International License (http://creativecommons. org/licenses/by/4.0/), which permits use, sharing, adaptation, distribution and reproduction in any medium or format, as long as you give appropriate credit to the original author(s) and the source, provide a link to the Creative Commons license and indicate if changes were made.

The images or other third party material in this chapter are included in the chapter's Creative Commons license, unless indicated otherwise in a credit line to the material. If material is not included in the chapter's Creative Commons license and your intended use is not permitted by statutory regulation or exceeds the permitted use, you will need to obtain permission directly from the copyright holder. 